\title{
Image Reconstruction Algorithm for Motion Insensitive Magnetic Resonance Fingerprinting (MRF): MORF
}

Bhairav Bipin Mehta ${ }^{1}$, Dan Ma ${ }^{1}$, Eric Yann Pierre ${ }^{2}$, Yun Jiang ${ }^{1}$, Simone Coppo ${ }^{1}$, and Mark Alan Griswold ${ }^{1,3}$

${ }^{1}$ Department of Radiology, Case Western Reserve University, Cleveland, Ohio, United States

${ }^{2}$ Imaging Division, The Florey Institute of Neuroscience and Mental Health, Melbourne, Victoria, Australia

${ }^{3}$ Department of Biomedical Engineering, Case Western Reserve University, Cleveland, Ohio, United States

To be submitted to Magnetic Resonance in Medicine as a Full Paper

Correspondence:

Mark Alan Griswold, Ph.D.

\section{Case Western Reserve University}

This is the author manuscript accepted for publication and has undergone full peer review but has not been through the copyediting, typesetting, pagination and proofreading process, which may lead to differences between this version and the Version of record. Please cite this article as doi:10.1002/ mrm.27227. 
11100 Euclid Ave - Bolwell B121

Cleveland, $\mathrm{OH} 44106$, USA

Email: mark.griswold@case.edu

Phone: +1-216-844-8085

Running Title: MOtion insensitive magnetic Resonance Fingerprinting (MORF)

Key Words: MR Fingerprinting, motion compensation, motion estimation, quantitative imaging, relaxation time, pattern recognition.

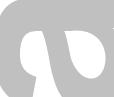

Word count: 5727 , figure count: 9 , table count: 1

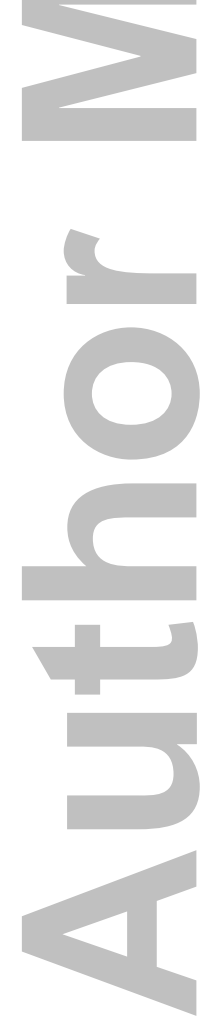




\section{Abstract}

\section{(O)}

Purpose: The purpose of this study is to increase the robustness of Magnetic Resonance Fingerprinting (MRF) towards subject motion.

Methods: A novel reconstruction algorithm, MOtion insensitive magnetic Resonance Fingerprinting (MORF), was developed, which employs an iterative reconstruction based retrospective motion correction approach. Each iteration loops through the following steps: pattern recognition, metric based identification of motion corrupted frames, registration based motion estimation, and motion compensated data consistency verification. The proposed algorithm was validated using in vivo 2D brain MRF data with retrospective in-plane motion introduced at different stages of the acquisition. The validation was performed using qualitative and quantitative comparisons between results from MORF, the iterative multi-scale (IMS) algorithm, and with the IMS results using data without motion for a ground truth comparison. Additionally, the MORF algorithm was evaluated in prospectively motion corrupted in vivo 2D brain MRF datasets.

Results: For datasets corrupted by in-plane motion both prospectively and retrospectively, MORF noticeably reduced motion artifacts compared to IMS and closely resembled the results from data without motion, even when $\sim 54 \%$ of data was motion corrupted during different parts of the acquisition.

Conclusion: MORF improves the insensitivity of MRF towards rigid-body motion occurring during any part of the MRF acquisition. 


\section{Introduction}

Patient motion is ubiquitous in clinical magnetic resonance imaging (MRI), and presents one of the biggest challenges in the field. In elderly or pediatric(1) patient populations, it is challenging to eliminate subject motion. Additionally, tremors are present in patients with neuro-degenerative diseases such as Parkinson's disease. Various practical measures are taken to reduce and eliminate subject motion such as fixation of the head using cushions or use of anesthesia. Cushion based restriction causes patient discomfort and cannot completely eliminate motion, while anesthesia present safety concerns and is not cost effective. The susceptibility of MR acquisition to patient motion can lead to a lengthened scan time or an error or loss in valuable diagnostic and therapeutic information. Lengthening the procedure can present a financial burden, whereas any errors or loss of clinical information can affect the patient outcome(2). Therefore, motion sensitivity of the MR acquisition can both reduce the value of the exam as well as reduce the quality of patient care.

Magnetic resonance fingerprinting $(\mathrm{MRF})(3)$ is a recently developed multi-parametric MRI framework. In this approach, various degrees of freedom in acquisition parameters are exploited by varying them in a pseudo-random fashion to generate unique signal evolutions, acting as fingerprints of the underlying tissue, that are specific to the tissue properties such as T1, T2, etc. This framework is analogous to and is inspired by conventional bio-metric fingerprinting. Using the Bloch equations and acquisition parameters, a bank of simulated signal evolutions, called the dictionary, is generated for various combinations of feasible tissue parameters. For reconstruction, signal 
evolutions from each spatial location are compared with all the simulated fingerprints within the dictionary and the best match is identified using a pattern recognition algorithm. The best matched fingerprint provides the underlying tissue and system parameters such as $\mathrm{T} 1, \mathrm{~T} 2, \mathrm{~B} 0$, etc. for the corresponding spatial location. It has been shown that MRF is highly efficient in estimating multiple quantitative tissue properties such as T1, T2, $(3,4)$ and has shown the potential of estimating perfusion(5-7), diffusion(8,9), exchange $(10,11)$, and system properties like $B 0(3)$ and $B 1+(12,13)$. Additionally, the use of pattern recognition for reconstruction along with the uniqueness of the signal evolutions provide a certain degree of error tolerance similar to what we see in the traditional biometric fingerprinting. It has been previously shown that MRF presents some insensitivity to subject motion(3), primarily towards motion present in the later stages of the acquisition. However, if substantial motion is present in the early stages of the acquisition then the previously proposed reconstruction techniques fail to recover parameter maps free of artifacts.

A significant amount of work has been done over the past 30 years to eliminate motion artifacts in brain imaging(14-16). However, it still presents an unsolved problem primarily because of the complexity of the issue. It has been widely accepted that there is no effective single solution which can be applied to most clinical situations(14-16). There are techniques which are extremely effective, but are limited by their applicability to specific situations. For example, camera based approaches require interaction with patients for placement of markers and cannot capture intrinsic motion such as cardiac motion. 
In this work we make a fresh attempt to tackle the motion issue. We propose an image reconstruction algorithm to improve upon the work done by Ma et al. (3) to further increase the robustness of MRF towards subject motion occurred during any part of the acquisition. In general, motion correction techniques for MRI are divided into prospective and retrospective motion correction. In general, the prospective motion correction approaches estimate the subject motion and adapt the acquisition in real-time such that the acquired data follows the desired object of interest. For these approaches, the subject motion information is mostly estimated through MR navigators(17-21) or external tracking devices(22-27). For retrospective approaches, the acquisition is performed without any real-time adjustments, however, the effects of motion are undone through modification of the collected data and/or the reconstruction model(28-32). Recently, apart from our work there have been several other retrospective motion correction approaches proposed to improve MRF's insensitivity towards motion. In general, these methods can be distributed into two classes. The first class contains approaches that change only the pattern recognition step $(33,34)$. The second class of methods(35-37) use a non-iterative multi step approach which uses motion estimation and compensation of the frames followed by pattern recognition. In this study, we will be employing a retrospective motion correction based approach in a form of iterative reconstruction method. The proposed method is evaluated in retrospectively and prospectively in-plane motion corrupted two dimensional (2D) human brain MRF data. Since the proposed method uses retrospective motion correction approach with motion information extracted from a 2D MRF acquisition without any special navigators for 
through-plane motion, the current implementation of the proposed approach is only capable to correct for in-plane motion.

\section{Methods}

\section{Algorithm Overview}

The proposed algorithm, MOtion insensitive magnetic Resonance Fingerprinting (MORF), is summarized in the flow chart in figure 1. First, pattern recognition is performed by projection of the acquired signal evolutions onto the dictionary using template matching. Next, motion corrupted frames (2D images corresponding to individual time-points) are identified based on the results from the pattern recognition. In the third step, displacement fields are estimated for the motion corrupted frames. Using these updated displacement fields, data consistency is verified in step four. Finally, pattern recognition is performed by projecting the updated data onto the dictionary. Steps 2 to 5 are repeated until convergence is met, which is measured using the relative norm of the update. Steps 2 and 3 are only executed for odd iterations to allow data stabilization for a given set of motion parameters. The output of the latest pattern recognition operation is used as the final result.

Pattern recognition: This operation is performed in similar fashion as previously described in Ma et al(3). Temporal signal evolutions from each spatial location are compared with all the simulated fingerprints within the dictionary. The comparison is performed using correlation measurement estimated from absolute of the complex inner 
product between normalized (unit $l_{2}$ norm) voxel signal evolution and the normalized simulated fingerprint. The simulated dictionary fingerprint with the highest correlation is considered as the best representation of the underlying signal and the corresponding tissue parameters used for the simulation are attributed to the respective voxel location. The relative spin density (M0) is computed as the scaling factor between the voxel signal evolution and the simulated fingerprint. This step replaces the signal evolution data at each spatial location with the matched fingerprint scaled by the respective M0. From here on, this data with each spatial location containing MO scaled matched simulated fingerprint is referred to as the data projected onto the dictionary, which will be used in operations involved in other blocks of the algorithm. Since the pattern recognition process provides the most consistent state across the frames, the data projected onto the dictionary is considered to be in most feasible co-registered state from the overall algorithm perspective.

Identification of motion corrupted frames: Figure 2 summarizes the process of identification of motion corrupted frames. For this step, temporally smoothed data before the pattern recognition process is considered as the reference data (Fig. S1). The temporal smoothing is performed using data view sharing with a window size of 48 for the first iteration and 3 for the remaining. Since the sensitivity of T1 and T2 varies across the acquisition, motion during different parts of the acquisition will appear differently in the T1 and T2 maps (Fig. 2). Thus, identification of motion corrupted frames is performed using three metrics (Fig. 2). The first metric is the normalized mutual information (NMI)(38) measured between the current estimated T1 map and the magnitude value of each frame of the reference data. Similarly, the second metric is the 
same except using the T2 map instead of T1. The third metric is the relative root mean square error (RMSE) between the reference data and the data projected onto the dictionary. For each of the three metrics, the entire set of frames is divided into two sections using an automatic threshold(39). The primary goal of the automatic thresholding approach is to distribute the frames into two sections only based on the metric value. To this end, Otsu's method(39) is a non-parametric and unsupervised thresholding approach which minimizes intraclass variance of the two sections. Additionally, Otsu's thresholding(39) algorithm is one of the most widely used automatic thresholding approaches with successful application to a wide variety of scenarios(40). The section with the smaller number of frames is considered as motion corrupted. For a given metric, if the number of identified motion corrupted frames is greater than one third (heuristically chosen value) of the total number of frames, then the identified frames are discarded for that specific metric only. The rationale behind this discarding step is that if a given metric does not present motion related information then the two sections after thresholding will be evenly distributed. An example situation is illustrated in figure 2, where NMI metric measured with the T1 map provides an even distribution. Additional example situations are shown in supporting figures S2, S3 and S4, which illustrate the advantages and disadvantages of each of the three metrics. Finally, the motion corrupted flag (1: motion corrupted; 0: motion free) identified using the three metrics are unified using a logical 'or' operation and the results are passed on to the motion estimation step.

Motion estimation: The motion estimation is performed for all the identified motion corrupted frames except for the frames corresponding to zero flip angle. The estimation 
of the rigid-body displacement fields are performed using image registration. For the image registration in this step, the magnitude value of both the input frames was used. The built-in functions in Matlab 2014b (Mathworks, Natick, MA) are used for image registration with Mattes mutual information $(41,42)$ as the registration metric. The registration operation is performed with low-resolution estimates of motion corrupted frames as moving data and their corresponding frames from the data projected onto the dictionary as the fixed reference data. For the first iteration, the average of the nonmotion corrupted frames from the data projected onto the dictionary is used as the reference fixed object. The low-resolution estimate of each frame used in the registration is generated by applying a radially symmetric Hanning window kernel to the motion-compensated (based on the motion estimates from previous iterations) k-space measurements. The windowing kernel parameters are: accept band $=10 \%$, transition band $=10 \%$ and stop band $=80 \%$. Additionally, to further mitigate the effects of undersampling artifacts, view-sharing with a window size of three is used. However, view-sharing leads to temporal blurring, thus, view-sharing is used only for the initial two iterations while a conjugate gradient based SENSE(43) operation is applied for the remainder of the iterations.

Data consistency is performed independently frame by frame using the following equation:

$$
\boldsymbol{m}^{*}=\boldsymbol{m}+\mathcal{F}_{\mathrm{u}}{ }^{-1} \mathcal{R}^{-1} \mathbf{d}-\mathcal{F}_{u}^{-1} \mathcal{F}_{\mathrm{u}} \boldsymbol{m} \quad \ldots[1]
$$

where $\boldsymbol{m}$ is the input frame, $\mathcal{R}$ is the motion registration operator, $\mathbf{d}$ is the measured kspace data, $\mathcal{F}_{\mathbf{u}}$ is the undersampled nuFFT(44) combined with normalized conjugate coil combination operation(43). The registration operator, $\mathcal{R}$, applies the estimated rigid- 
body motion to complex valued k-space data. Conceptually, $\boldsymbol{m}$ represents the current estimate of the frame in the co-registered state in spatial domain, while $\mathbf{d}$ is the acquired undersampled k-space data for the corresponding time point. The second term in the equation represents the co-registered equivalent of the measured data in the spatial domain. The third term is the undersampled equivalent of $\boldsymbol{m}$. The output $\boldsymbol{m}^{*}$ is fed to the pattern recognition step.

\section{Retrospectively simulated motion experiments.}

The proposed MORF algorithm was first evaluated in experiments with retrospectively simulated motion where the ground truth was known. To closely resemble the realistic conditions, a fully sampled (48 spiral arms) in vivo brain data was acquired in a healthy subject using a FISP based MRF acquisition(4). Two different motion patterns, which are described in detail below, were introduced to the coil-combined MRF frames of the fully sampled brain data using MATLAB functions from the image processing toolbox (Mathworks, Natick, MA). In order to evaluate the robustness of the algorithm, two sets of simulations corresponding to each motion pattern were performed. For each of these simulation sets, multiple datasets were generated with the simulated motion introduced at different parts of the acquisition. Specifically, each set of simulation experiments was comprised of eight datasets, each with 30\% (300 frames out of 1000 frames) of the data being motion corrupted and the position of the motion being shifted by an interval of 100 frames from one data set to the other. The motion-corrupted frames for each dataset were multiplied by simulated coil maps to generate multi-channel data. These motion-corrupted multi-channel frames were forward sampled for a single spiral arm 
using non-uniform fast Fourier transform (nuFFT)(44) to estimate the undersampled kspace measurements, which were used as inputs in the reconstruction algorithm.

Both the motion pattern simulates a back and forth head motion in the left-right direction (yaw). However, the first motion pattern used represents a single rotation of the subject's head about the yaw axis followed by a return to the original position (referred to as 'single motion'). In this pattern the head position gradually rotates from the original position $\left(0^{\circ}\right)$ to $45^{\circ}$ with respect to the original position, where it stays for a while and then the head gradually rotates back to the original position $\left(0^{0}\right)$. The second motion pattern represents multiple back and forth yaw of the head (referred to as 'oscillatory motion') with a smaller range of motion. In this pattern the head continuously rotates along the left-right direction with the maximum rotation of $\pm 15^{0}$ with respect to the original position.

Throughout this study, the iterative multi-scale (IMS) algorithm(45) was used as the reference reconstruction algorithm for MRF data. In order to see effects primarily from motion and not from other effects such as retrospective non-Cartesian data undersampling, we used a reference data that retains the same processing steps. Thus, apart from the two motion patterns, an undersampled data was generated free from simulated motion which was reconstructed using the IMS algorithm. These results were considered as the ground truth for both simulation experiments. For evaluation of the mapping results, qualitative comparison was performed visually while quantitative comparison was performed using the structural similarity (SSIM) index(46) and relative root-mean square error (rRMSE), which is calculated using following equation: 


$$
r R M S E=\frac{\sqrt{\sum_{i \in R O I}\left(\theta_{i}-\theta^{g t}\right)^{2}}}{\sqrt{\sum_{i \in R O I}\left(\theta^{g t}\right)_{i}^{2}}} \times 100
$$

where $\theta_{i}$ is the estimated parameter at location $i, \theta^{g t}$ is the ground truth value of the parameter, and $i$ covers the entire region of interest, which in this study is the entire brain from the gold standard data.

\section{In vivo experiments with motion}

Finally the proposed reconstruction algorithm was evaluated in vivo with intentionally motion-corrupted brain imaging data. A healthy volunteer was requested to move their head back and forth in the left-right direction (yaw) during a FISP based 2D MRF acquisition(4). Acquisitions with two types of timing for subject motion were performed. The first one was with motion requested in the initial $\sim 3 \mathrm{~s}(\sim 210$ frames; $22 \%)$ of a $13.5 \mathrm{~s}$ (1000 frames) acquisition. The communication with the subject was performed by playing music through the scanner headset (music on $=$ the subject has to move the head; music off $=$ the subject has to stay still) to limit possible communication errors. For the second acquisition, the subject was allowed to move during random parts of the acquisition according to his/her will. A separate acquisition without subject motion was acquired as a reference measurement. For quantitative evaluation, T1 and T2 values measured over a manually drawn region of interest (ROI) for gray matter (frontal region) and white matter (frontal and parietal regions) were compared between MORF results and the results from data without motion. Additionally, a conventional T2 weighted turbo spin echo (TSE) acquisition was performed with motion equivalent to the first acquisition ( $14.3 \mathrm{sec} ; 21 \%$ motion corrupted from a total duration of $68 \mathrm{sec})$. For comparison, 
synthetic T2 weighted images were generated from maps reconstructed using MORF algorithm from data with intentional motion.

\section{Acquisition and reconstruction details}

All imaging was performed on a 3T (Skyra, Siemens) scanner and in accordance with the protocols approved by our institutional review board (IRB). For this study, a FISP based $\operatorname{MRF}(4)$ acquisition was used to perform 2D brain imaging. A standard 16 channel head coil was used for signal reception. The following imaging parameters were used for the study: FOV $=300 \times 300 \mathrm{~mm}^{2}$, matrix $=256 \times 256$, single slice with thickness $=5 \mathrm{~mm}$, minimum TR $=12 \mathrm{~ms}, \mathrm{TE}=1.9 \mathrm{~ms}$ and total number of frames $=1000$. For k-space measurement, a single-shot dual-density spiral trajectory $(47,48)$ was used with a bit-reverse reordering along the temporal dimension to reduce the view sharing kernel window size. With respect to the Nyquist sampling rate, the inner region of the trajectory had an undersampling rate $R=3$ for a matrix size of $20 \times 20$ and the outer region had $R=48$ at the edge of k-space. The acquisition parameters of the T2 weighted TSE scan were as follows: FOV $=230 \times 187 \mathrm{~mm}^{2}$, matrix $=320 \times 198$, slices $=$ 32, slice thickness $=5 \mathrm{~mm}, \mathrm{TR}=5650 \mathrm{~ms}, \mathrm{TE}=94 \mathrm{~ms}$, refocusing $\mathrm{FA}=150^{\circ}$ and turbo factor $=18$. The T2 weighted synthetic image were generated using following equation

$$
T 2 w \text { signal }=M 0 *\left(1-e^{\left(-\frac{T R}{T 1}\right)}\right) * e^{-T E / T 2} \ldots[3]
$$

with $\mathrm{TR}=5650 \mathrm{~ms}$ and $\mathrm{TE}=50 \mathrm{~ms}$.

A dictionary based on the sequence parameters was simulated using the extended phase graph formalism(4). The T1 and T2 value ranges were selected based on the 
expected physiological ranges for brain imaging. Specifically, the T1 values ranged from $60 \mathrm{~ms}$ to $2000 \mathrm{~ms}$ with a step size of $10 \mathrm{~ms}$, from $2020 \mathrm{~ms}$ to $3000 \mathrm{~ms}$ with a step size $20 \mathrm{~ms}$, from $3050 \mathrm{~ms}$ to $3500 \mathrm{~ms}$ with a step size of $50 \mathrm{~ms}$ and from $4000 \mathrm{~ms}$ to $5000 \mathrm{~ms}$ with a step size of $500 \mathrm{~ms}$. The T2 values ranged from $10 \mathrm{~ms}$ to $200 \mathrm{~ms}$ with a step size of $5 \mathrm{~ms}$, from $210 \mathrm{~ms}$ to $300 \mathrm{~ms}$ with a step size of $10 \mathrm{~ms}$ and from $350 \mathrm{~ms}$ to $500 \mathrm{~ms}$ with a step size of $50 \mathrm{~ms}$.

MORF was implemented in a MATLAB (Mathworks, Natick, MA) based script. Additionally, this work made use of the High Performance Computing Resource in the Core Facility for Advanced Research Computing at Case Western Reserve University. The current implementation used functions or modified version of functions from Prof. Jeffrey Fessler's iterative reconstruction toolbox(49) for nuFFT related operations, from Andriy Myronenko's medical image registration toolbox(50) for interpolation related operations, and from Adam Pocock's MIToolbox(51) for mutual information based operations.

\section{Results}

\section{Retrospectively simulated motion experiments}

Figure 3 shows an example comparison of the MORF results with the reconstruction results using IMS for data with simulated single motion pattern in the beginning of the acquisition and IMS reconstruction results using data without motion as a reference. For IMS (second column), the head position in the T1 map is completely different than the 
one in the T2 map. Interestingly, the T1 map appears to retain many of the structures in the central region, however, the peripheral area is heavily corrupted by motion artifacts thereby rendering it unusable. The T2 map retains the head position similar to the reference results, but presents a severe amount of motion artifacts. On the other hand, both T1 and T2 maps using MORF closely resemble the reference maps and present minimal residual errors. Figure 4 shows example mapping results comparing MORF with IMS and the ground truth for the simulated oscillatory motion pattern in the beginning of the acquisition. For this motion pattern, the T1 map using IMS presents severe motion artifacts. However, the corresponding T2 map presents fewer errors compared to T1 map. Again, both T1 and T2 maps using MORF closely resemble the reference maps and present minimal errors. The accuracy of the estimation of the three rigid-body based motion parameters using MORF algorithm for data with simulated oscillatory motion pattern is shown in figure 5 . The estimated rotation values are in close agreement with the ground truth. The most pronounced mismatch in the estimation of the rotation angle is for frames 201 to 210 because those frames correspond to the acquisition segment with zero flip angle (green blocks in figure 5), where estimation of motion is not performed due to low SNR. On the other hand, the translation measurements present small variations. Overall, the estimated motion parameters closely match the ground truth with absolute error under 1.25 pixel for translation and 0.88 degrees for rotation (excluding the frames corresponding to zero flip angle).

Figure 6 and 7 show the quantitative results from both sets of simulations with single and oscillatory head yaw motion patterns, respectively. We see the rRMSE and SSIM 
values using the results from MORF algorithm consistently outperforms the results from IMS algorithm. Additionally, we see the proposed method performs consistently with respect to motion occurring at different stages of the acquisitions and with different pattern of motion, illustrating its robustness. The quantitative results from IMS also illustrate that for FISP-MRF acquisition, the T1 map is primarily affected when motion occurs in the early stage of the acquisition while T2 map is affected when motion occurs in either early or middle stage of the acquisition.

\section{In vivo experiments with motion}

Figure 8 shows results from in vivo scans with subject motion during the initial $22 \%$ of the acquisition. The reconstructed T1 map using the IMS algorithm is corrupted by severe motion artifacts while the corresponding T2 map does not present any visible motion artifacts. The MORF algorithm successfully eliminates most of the visible motion artifacts and recovers many of the previously missing structures. The results of the MORF algorithm closely resemble the results from data without subject motion. Although, we still see some differences in structures between the MORF maps and reference maps which might be due to change in slice location caused by interscan motion as seen from the shape of the ventricles in the T2 maps (arrows in figure 8A). The average frame of the MRF data with motion illustrates the severity of the data corruption if the MRF data were treated as a conventional scan. The acquired T2 weighted TSE image (top row) illustrates the extent and the severity of the data corruption for comparable amount of subject motion in a conventional acquisition used clinically. On the other hand, the synthetic T2 weighted image (bottom row) generated 
from MORF results present minimal artifacts. For clarification, the T2 weighted TSE data is only shown to illustrate the severity of data corruption due to motion for a conventional clinical acquisition. Figure $8 \mathrm{~B}$ shows the estimated 2D rigid-body motion parameters using MORF algorithm.

Figure 9 shows results from in vivo scans with subject motion during random parts of the acquisition. As we see in the estimated motion parameters (Figure 9B), the subject ended up moving in the beginning $\sim 17 \%$ of the acquisition as well as at the ending $\sim 37 \%$ of the acquisition with a total of $\sim 54 \%$ data ( $\sim 540$ frames out of 1000 frames) being motion corrupted. The sharp jumps in estimated motion parameters occur for frames near zero flip angle due to low SNR. Figure 9A shows the reconstruction results of the corresponding experiment. Again, IMS results present severe motion artifacts not only in the T1 map but also in the T2 map, which are significantly reduced in MORF results. Additionally, MORF results closely resemble the results from data without motion with a few structural differences which might be due to difference in slice position due to interscan motion.

Table 1 lists the estimated ROI based T1 and T2 values for the brain tissues from all the in vivo results. We see a close agreement between MORF results using data with motion and the reference results (IMS without motion).

\section{Discussion}

In this study we have presented a reconstruction algorithm to retrospectively improve the motion insensitivity of MRF acquisition. The proposed method, MORF, was 
validated using simulations with retrospective addition of $2 \mathrm{D}$ in-plane motion where ground truth was known. Our simulation results show that the MORF algorithm consistently provides results in close agreement to the ground truth for motion present at different stages of the acquisition as well as for different motion paradigms. Finally, we illustrated the performance of MORF in prospectively motion corrupted in vivo datasets. In the in vivo experiments, we showed that MORF results are in close agreement with reference results from data without motion for data with $22 \%$ of motion corruption in the beginning of the acquisition and for data with $54 \%$ of motion corruption during different parts of the acquisition.

As mentioned above, there have been several other reconstruction approaches proposed to improve MRF's insensitivity towards motion. The first class contains approaches that change only the pattern recognition step wherein the motion corrupted frames are either discarded(34) or adaptively weighted using a robust-fitting approach(33). However, discarding frames would lead to a loss of potentially useful information, which is avoided to a certain extent in the proposed approach. The second class of approaches(35-37) in general use following steps in their algorithm: 1. Reduction of aliasing artifacts in MRF frames, 2. Image registration based motion estimation, 3. Generation of motion compensated frames and 4. Pattern recognition. For reduction of aliasing artifacts, either a traditional sliding window $(35,36)$ or a softweighted key-hole(37) approach is used in these methods. The primary difference with MORF is that it iterates back and forth between pattern recognition and motion estimation/compensation steps, wherein conceptually each of those step is helping the other step to drive the solution towards motion artifact free result. As it was previously 
shown, MRF inherently provides a certain degree of tolerance to error through the use of unique signal evolutions and pattern recognition(3). This feature of partial insensitivity to errors allows MRF to achieve high acceleration rates and some tolerance to motion(3). The MORF algorithm exploits this feature of MRF to further improve upon its robustness to subject motion. The iterative approach would reduce the restrictions on the accuracy and robustness of the sub-components, although, at the expense of computation time. It is worth noting that, even if the motion identification and motion estimation steps do not exactly match the simulated input motion, as seen in figure 5 , the output maps show a high level of agreement with the ground truth. This is primarily due to the inherent robustness of MRF to errors.

Figure 6 and 7 show different parts of the acquisition present different sensitivities to T1 and T2 errors. Although MRF intends to spread the information of all the parameters evenly throughout the acquisition, in practice each time point is relatively more sensitive to one parameter over the others, thereby, significantly affecting the corresponding parameter map. The variant of MRF acquisition scheme used in this study starts the acquisition with an inversion pulse, thus, the initial time points heavily contribute to T1 related information while the time points close to high FA lobe heavily contribute to T2 related information. This variation in parameter sensitivity also explains the results in the middle column of figure 3 , where the T1 map appears to be rotated while T2 map is approximately straight. Please note that MRF, in general, presents many degrees of freedom in manipulating the acquisition, and these must be considered when performing motion correction. 
The primary focus of the current work was to improve the motion insensitivity of MRF. However, the proposed MORF algorithm also provides displacement fields and can be used for motion estimation which would be potentially valuable for a variety of applications such as cardiac(52) and abdominal imaging $(53,54)$. In this study, we have presented one approach for performing motion identification and motion estimation. However, the MORF algorithm is not limited to these approaches and can be used with a wide variety of methods for motion identification and estimation based on the required application. Moreover, the general idea can potentially be applied to any phenomenon which leads to model errors. Additionally, the MORF algorithm is flexible enough such that it can be merged with other constraint based iterative reconstruction approaches(45,55-60) applied to MRF.

The current implementation of the MORF algorithm has a few limitations. As with most of the iterative based reconstruction algorithms, MORF is also computationally intensive and presents a relatively long reconstruction time for clinical use. Currently, MORF is implemented in a MATLAB (Mathworks, Natick, MA) based script which uses our university's high performance computing cluster to speed up the reconstruction. The reconstruction time for a typical dataset is around 1 hour, which varies depending on the availability of the cluster nodes. The reconstruction speed can be further improved by making use of higher level languages such as $\mathrm{C} / \mathrm{C}++$ and advanced hardware such as Graphical Processing Units. Additionally, a reconstruction framework such as Gadgetron(61) would be valuable to make it feasible for the clinical environment.

For a $2 \mathrm{D}$ brain acquisition, motion can be in-plane and/or through-plane. Another limitation of the current implementation is the inability to correct for the effects of 
through-plane motion. However, this limitation is primarily dependent on the 2D nature of the acquisition and is a limitation common to all $2 \mathrm{D}$ retrospective motion correction based methods. Through-plane motion changes the spin history compared to in-plane or no motion and causes differences in the signal evolutions. For a conventional MR imaging scan this would, in general, lead to an artifact. However, the ramifications of such changes in signal evolutions are complicated for MRF. Depending on the fidelity and uniqueness of the uncorrupted segment of the signal evolution, the pattern recognition algorithm might still be able to "see through the errors" and properly match the corrupted signal evolution to the correct dictionary entry. This is dependent on many factors such as the amount of motion, when the motion happens within the acquisition, the specific MRF sequence parameters, etc. One such example study is presented by Yu et al. (34), that evaluates the effects of through-plane, in-plane and a combination of it at three different locations (start, middle and end of acquisition) in a FISP based MRF acquisition. Their results show that the $\mathrm{T} 1$ map is primarily affected by in-plane motion in the beginning of the scan with motion resulting in blurring. As for the T2 map, their results show in-plane motion in the beginning or middle of the acquisition results in blurring, while through-plane motion in the middle of the acquisition results in underestimation of T2. On the other hand, since the limitation associated with throughplane motion is primarily due to the $2 \mathrm{D}$ spatial coverage nature of the acquisition and is not of a concern for a $3 \mathrm{D}$ spatial coverage acquisition, the issue can be avoided by using an acquisition with 3D spatial coverage instead of 2D coverage. The approach presented in this work is an initial step towards a long-term end goal of eliminating all types of motion in a 3D acquisition. The MORF approach can potentially be applied to a 
3D volumetric acquisition. Recently, MRF was extended to perform 3D spatial coverage $(54,62-64)$. A future step would be the extension and characterization of the MORF algorithm with the 3D MRF acquisition.

The present implementation of MORF is also limited to rigid-body motion, thereby confining the application of MORF to systems presenting only rigid-body motion. The extension of MORF to non-rigid motion would be valuable for applications like cardiac and abdominal MRF, where it would not only reduce the acquisition time and increase the scan efficiency, but also provide displacement based motion information. However, estimation of non-rigid displacement fields is challenging especially when working on frames accelerated by a factor of 48 . In the current MORF implementation, no prior information or adaptive filtering was used for displacement fields. However, spatiotemporal prior information could be exploited to improve the accuracy of the estimation of the displacement fields(65). The use of displacement based spatio-temporal information in MORF algorithm could be explored in future to overcome the challenge of non-rigid body motion.

For experiments with simulated retrospective in-plane motion, the reference results were used from retrospectively undersampled data as opposed to fully sampled data. As mentioned above, this reference data retains the same processing steps and was used to see effects primarily from motion and not from other effects such as retrospective non-Cartesian data undersampling. However, due to absence of ideal ground truth the corresponding evaluation results could potentially present biases. Figure S5 shows mapping results comparing direct matching results of fully sampled and undersampled data and IMS results of undersampled data. 
The estimated T2 values from in vivo experiments (Table 1) are different from the literature values, however, the values are in agreement with the reference MRF data without motion. Work from our group has shown B1 related effects in part contribute to these differences(66). Furthermore, other groups have shown stimulated echoes(67), slice profile(68), magnetization transfer(69) and finite RF pulses(70) in steady state sequences affecting the T2 values as compared to spin echo acquisitions. While the exact source of these differences is not clear at this point, the exact quantification of these effects is ongoing research at multiple sites, and is beyond the scope of the current study.

Reduction of the motion sensitivity of MR acquisitions would not only help reduce the cost but also improve the throughput, which would be valuable for sectors with high demand. Additionally, the benefits of reduction in loss of clinical information are invaluable. Considering all the limitations of the proposed method and the fact that the acquisition time is only $13.5 \mathrm{~s}$, the preferred mode of operation would be to repeat the measurement. However, in situations where repetition is difficult or not possible, the proposed method would be valuable to retrieve clinical information from the existing motion inflicted acquisitions. MORF, in the future, can be combined with approaches like Music-MRF(62) to potentially reduce the use of anesthetization in pediatric cases, which would be immensely valuable to not only improve patient care but also reduce the healthcare cost. 


\section{Conclusions}

(l)

We proposed and validated a novel reconstruction algorithm which identifies and corrects motion corrupted frames not only to further increase the motion insensitivity of MRF but also to provide estimation of the in-plane motion parameters. The proposed algorithm was able to compensate effects of rigid-body motion for $2 \mathrm{D}$ brain imaging occurred during any part of the acquisition. We showed that the MORF algorithm successfully works even if $\sim 54 \%$ data is corrupted with motion occurring during different parts of the acquisition. 


\section{Figures}

Figure 1: Flow chart of the motion insensitive magnetic resonance fingerprinting (MORF) algorithm.

Figure 2: Steps involved in identification of motion corrupted frames. Motion corrupted frames are identified based on three metrics: Normalized mutual information (NMI) between each frame of the temporally smooth data and T1 map, NMI between each frame of the temporally smooth data and T2 map, and relative root mean square error (RMSE) between temporally smooth data and data projected onto the dictionary. Automatic thresholding is used to split each metric into two sections. The section with lower number frames is considered motion corrupted. For a given metric, if the number of identified motion corrupted frames are greater than one third of the total number of frames then those frames are discarded (top row). Finally, the results from the three metrics are combined using a logical OR operation to provide the motion flag (1: frame with motion ; 0: frame without motion) output.

Figure 3: Example mapping results from the simulated single motion pattern in the beginning of the acquisition. First column: maps using IMS reconstruction from data without simulated motion; Second column: maps using IMS reconstruction from data with simulated single motion pattern; Third column: maps using MORF reconstruction from data with simulated single motion pattern. First row: T1 maps; Second row: T2 maps; Third row: T1 error maps with first column as ground truth; Forth row: T2 error maps with first column as ground truth. IMS results present severe motion artifacts. However, MORF results closely resemble to the ground truth with minimal errors. 
Figure 4: Example mapping results from the simulated oscillatory motion pattern in the beginning of the acquisition. First column: maps using IMS reconstruction from data without simulated motion; Second column: maps using IMS reconstruction from data with simulated oscillatory motion pattern; Third column: maps using MORF reconstruction from data with simulated oscillatory motion pattern. First row: T1 maps; Second row: T2 maps; Third row: T1 error maps with first column as ground truth; Forth row: T2 error maps with first column as ground truth. Again, IMS results present motion artifacts which are significantly minimized in MORF results.

Figure 5: Accuracy of the estimation of rigid-body motion parameters. Example results from experiments with simulated oscillatory motion pattern in the beginning of the acquisition. top row: Translation along $\mathrm{X}$. middle row: Translation along $\mathrm{Y}$. bottom row: Rotation. The estimated rigid-body motion parameters are in close agreement with the ground truth values. The section highlighted with green blocks correspond to frames with zero flip angle where motion estimation is not performed.

Figure 6: Quantitative results from set of simulations with single motion pattern evaluating the performance of the reconstruction algorithms to motion occurring at different stages of the acquisition. First column: SSIM results; Second column: Relative RMSE results; First row: Results for T1 values; Second row: Results for T2 values. The variation in SSIM and rRMSE values for IMS algorithm illustrates the sensitivity of each parameter to motion for different parts of the acquisition. For MORF, the SSIM values are consistently high and the relative RMSE are consistently low, illustrating its agreement with ground truth for motion occurring at different stages of the acquisition. 
Figure 7: Quantitative results from set of simulations with oscillatory motion pattern evaluating the performance of the reconstruction algorithms to motion occurring at different stages of the acquisition. First column: SSIM results; Second column: Relative RMSE results; First row: Results for T1 values; Second row: Results for T2 values. Again, for all the datasets with motion during different parts of the acquisition, the SSIM and the relative RMSE values for MORF are consistently high and low, respectively.

Figure 8: Results from prospectively motion corrupted in vivo experiment with motion in the initial $\sim 22 \%$ of the acquisition.

A: Reconstruction results. First column: IMS reconstruction maps from a separate scan without subject motion; Second column: IMS reconstruction maps using data with subject motion; Third column: MORF reconstruction maps using data with subject motion; Fourth column: Temporally averaged frame of the raw MRF data with subject motion; Fifth column: conventional T2w-TSE. Top row is acquired data with equivalent subject motion and bottom row is synthetic image generated from maps reconstructed using MORF from data with motion. The results are consistent with simulated motion experiments. MORF noticeably increases the robustness of the MRF acquisition towards subject motion. It should be noted that the discrepancy in the brain structure between data with and without motion might be due to change in the imaging location because of inter-scan subject motion.

B: Estimated rigid-body motion parameters. top row: Translation along X; middle row: Translation along Y; bottom row: Rotation. 
Figure 9: Results from a prospectively motion corrupted in vivo experiment. For this experiment the subject was requested to move randomly according to his/her will.

A: Reconstruction results. First column: IMS reconstruction maps from a separate scan without subject motion; Second column: IMS reconstruction maps using data with subject motion; Third column: MORF reconstruction maps using data with subject motion; Fourth column: Temporally averaged frame of the raw MRF data with subject motion; Fifth column: Synthetic T2w-TSE image generated from maps reconstructed using MORF from data with motion. IMS results present a severe amount of motion artifacts not only in the T1 map but also in the T2 map. MORF results present significantly fewer artifacts compared to IMS and closely resemble the results from scan without motion.

B: Estimated rigid-body motion parameters. top row: Translation along $\mathrm{X}$; middle row: Translation along Y; bottom row: Rotation. The subject ended up moving in the beginning $\sim 17 \%$ of the acquisition as well as at the ending $\sim 37 \%$ of the acquisition with a total of $\sim 54 \%$ data being motion corrupted.

Supporting Figure S1: Origin of the components used for identification of motion corrupted frames.

Supporting Figure S2: Example metric curves illustrating usefulness of NMI with T2 map metric.

Supporting Figure S3: Example metric curves illustrating usefulness of NMI with T1 map metric.

Supporting Figure S4: Example metric curves illustrating usefulness of RMSE metric. 
Supporting Figure S5: Comparison of fully sampled data with undersampled data and direct matching with IMS algorithm. We see a few differences between results using direct matching from fully sampled data (left) and results using direct matching from undersampled data (center). However, results using direct matching and IMS algorithm (right) from undersampled data are in close agreement.

\section{Tables}

Table 1: ROI based T1 and T2 relaxation times from brain tissues measured across all the intentionally motion corrupted in vivo results.

\begin{tabular}{|c|c|c|c|c|}
\hline & \multicolumn{2}{|c|}{ T1 } & \multicolumn{2}{c|}{ T2 } \\
\hline Tissue & IMS without & MORF with & IMS without & MORF with \\
& motion & motion & motion & motion \\
\hline White matter & $833 \pm 55 \mathrm{~ms}$ & $849 \pm 70 \mathrm{~ms}$ & $41 \pm 5.7 \mathrm{~ms}$ & $41 \pm 6.1 \mathrm{~ms}$ \\
Gray matter & $1302 \pm 111 \mathrm{~ms}$ & $1333 \pm 112 \mathrm{~ms}$ & $66 \pm 8.4 \mathrm{~ms}$ & $61 \pm 6.5 \mathrm{~ms}$ \\
\hline
\end{tabular}




\section{References}

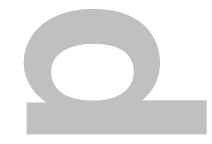

1. Brown TT, Kuperman JM, Erhart M, White NS, Roddey JC, Shankaranarayanan A, Han ET, Rettmann D, Dale AM. Prospective motion correction of high-resolution magnetic resonance imaging data in children. Neuroimage 2010;53:139-145. doi: 10.1016/j.neuroimage.2010.06.017.

2. Andre JB, Bresnahan BW, Mossa-Basha M, Hoff MN, Smith CP, Anzai Y, Cohen WA. Toward Quantifying the Prevalence, Severity, and Cost Associated With Patient Motion During Clinical MR Examinations. J. Am. Coll. Radiol. 2015;12:689-695. doi: 10.1016/j.jacr.2015.03.007.

3. Ma D, Gulani V, Seiberlich N, Liu K, Sunshine JL, Duerk JL, Griswold MA. Magnetic resonance fingerprinting. Nature 2013;495:187-92. doi: 10.1038/nature11971.

4. Jiang $Y$, Ma D, Seiberlich N, Gulani V, Griswold MA. MR fingerprinting using fast imaging with steady state precession (FISP) with spiral readout. Magn. Reson. Med. 2015;74:1621-1631. doi: 10.1002/mrm.25559.

5. Christen T, Pannetier NA, Ni WW, Qiu D, Moseley ME, Schuff N, Zaharchuk G. MR vascular fingerprinting: A new approach to compute cerebral blood volume, mean vessel radius, and oxygenation maps in the human brain. Neuroimage 2014;89:262270. doi: 10.1016/j.neuroimage.2013.11.052.

6. Wright KL, Ma D, Jiang Y, Gulani V, Griswold MA, Hernandez-Garcia L. Theoretical Framework for MR Fingerprinting with ASL: Simultaneous Quantification of CBF, Transit Time, and T1. Proc. Intl. Soc. Mag. Reson. Med 2014:417. 
7. Su P, Mao D, Liu P, Li Y, Pinho MC, Welch BG, Lu H. Multiparametric estimation of brain hemodynamics with MR fingerprinting ASL. Magn. Reson. Med. 2016. doi: 10.1002/mrm.26587.

8. Jiang Y, Hamilton JI, Lo W-C, Wright KL, Ma D, Coristine AJ, Seiberlich N, Gulani V, Griswold MA. Simultaneous T1, T2 and Diffusion Quantification using Multiple Contrast Prepared Magnetic Resonance Fingerprinting. Proc. Intl. Soc. Mag. Reson. Med 2017:1171.

9. Jiang Y, Ma D, Wright KL, Seiberlich N, Gulani V, Griswold MA. Simultaneous T1, T2, Diffusion and Proton Density Quantification with MR Fingerprinting. Proc. Intl. Soc. Mag. Reson. Med 2014:28.

10. Hamilton JI, Deshmane A, Hougen S, Griswold M, Seiberlich N. Magnetic Resonance Fingerprinting with Chemical Exchange (MRF-X) for Quantification of Subvoxel T1, T2, Volume Fraction, and Exchange Rate. Proc. Intl. Soc. Mag. Reson. Med 2015:329.

11. Hamilton JI, Griswold MA, Seiberlich N. MR Fingerprinting with chemical exchange (MRF-X) to quantify subvoxel T1 and extracellular volume fraction. J. Cardiovasc. Magn. Reson. 2015;17(Suppl 1):W35. doi: 10.1186/1532-429X-17-S1-W35.

12. Buonincontri G, Sawiak SJ. MR fingerprinting with simultaneous B1 estimation. Magn. Reson. Med. 2016;76:1127-1135. doi: 10.1002/mrm.26009.

13. Cloos MA, Knoll F, Zhao T, Block KT, Bruno M, Wiggins GC, Sodickson DK. Multiparametric imaging with heterogeneous radiofrequency fields. Nat. Commun. 2016;7:12445. doi: 10.1038/ncomms12445. 
14. Maclaren J, Herbst M, Speck O, Zaitsev M. Prospective motion correction in brain imaging: A review. Magn. Reson. Med. 2013;69:621-636. doi: 10.1002/mrm.24314.

15. Zaitsev M, Maclaren J, Herbst M. Motion artifacts in MRI: A complex problem with many partial solutions. J. Magn. Reson. Imaging 2015;42:887-901. doi: 10.1002/jmri.24850.

16. Godenschweger F, Kägebein U, Stucht D, Yarach U, Sciarra A, Yakupov R, Lüsebrink F, Schulze P, Speck O. Motion correction in MRI of the brain. Phys. Med. Biol. 2016;61:R32-R56. doi: 10.1088/0031-9155/61/5/R32.

17. White N, Roddey C, Shankaranarayanan A, Han E, Rettmann D, Santos J, Kuperman J, Dale A. PROMO: Real-time prospective motion correction in MRI using image-based tracking. Magn. Reson. Med. 2010;63:91-105. doi: 10.1002/mrm.22176.

18. Thesen S, Heid O, Mueller E, Schad LR. Prospective acquisition correction for head motion with image-based tracking for real-time fMRI. Magn. Reson. Med. 2000;44:457465. doi: 10.1002/1522-2594(200009)44:3<457::AID-MRM17>3.0.CO;2-R.

19. Fu ZW, Wang Y, Grimm RC, Rossman PJ, Felmlee JP, Riederer SJ, Ehman RL. Orbital navigator echoes for motion measurements in magnetic resonance imaging. Magn. Reson. Med. 1995;34:746-753. doi: 10.1002/mrm.1910340514.

20. Welch EB, Manduca A, Grimm RC, Ward HA, Jack Jr. CR. Spherical navigator echoes for full 3D rigid body motion measurement in MRI. Magn. Reson. Med. 2002;47:32-41. doi: 10.1002/mrm.10012.

21. van der Kouwe AJW, Benner T, Dale AM. Real-time rigid body motion correction 
and shimming using cloverleaf navigators. Magn. Reson. Med. 2006;56:1019-1032. doi: $10.1002 / \mathrm{mrm} .21038$.

22. Korin HW, Felmlee JP, Riederer SJ, Ehman RL. Spatial-Frequency-Tuned Markers and Adaptive Correction for Rotational Motion. Magn. Reson. Med. 1995;33:663-669. doi: $10.1002 / \mathrm{mrm} .1910330511$.

23. Sengupta S, Tadanki S, Gore JC, Welch EB. Prospective real-time head motion correction using inductively coupled wireless NMR probes. Magn. Reson. Med. 2014;72:971-985. doi: 10.1002/mrm.25001.

24. Zaitsev M, Dold C, Sakas G, Hennig J, Speck O. Magnetic resonance imaging of freely moving objects: prospective real-time motion correction using an external optical motion tracking system. Neuroimage 2006;31:1038-1050. doi: 10.1016/J.NEUROIMAGE.2006.01.039.

25. Ooi MB, Krueger S, Thomas WJ, Swaminathan S V., Brown TR. Prospective realtime correction for arbitrary head motion using active markers. Magn. Reson. Med. 2009;62:943-954. doi: 10.1002/mrm.22082.

26. Maclaren J, Armstrong BSR, Barrows RT, et al. Measurement and Correction of Microscopic Head Motion during Magnetic Resonance Imaging of the Brain Hess CP, editor. PLoS One 2012;7:e48088. doi: 10.1371/journal.pone.0048088.

27. Schulz J, Siegert T, Reimer E, Labadie C, Maclaren J, Herbst M, Zaitsev M, Turner R. An embedded optical tracking system for motion-corrected magnetic resonance imaging at 7T. Magn. Reson. Mater. Physics, Biol. Med. 2012;25:443-453. doi: 10.1007/s10334-012-0320-0. 
28. Atkinson D, Hill DLG, Stoyle PNR, Summers PE, Clare S, Bowtell R, Keevil SF. Automatic compensation of motion artifacts in MRI. Magn. Reson. Med. 1999;41:163170. doi: 10.1002/(SICI)1522-2594(199901)41:1<163::AID-MRM23>3.0.CO;2-9.

29. McGee KP, Felmlee JP, Jack CR, Manduca A, Riederer SJ, Ehman RL. Autocorrection of Three-Dimensional Time-of-Flight MR Angiography of the Circle of Willis. Am. J. Roentgenol. 2001;176:513-518. doi: 10.2214/ajr.176.2.1760513.

30. Leung G, Plewes DB. Retrospective motion compensation using variable-density spiral trajectories. J. Magn. Reson. Imaging 2005;22:373-380. doi: 10.1002/jmri.20388.

31. Louis Lauzon M, Rutt BK. Generalized K-space analysis and correction of motion effects in MR imaging. Magn. Reson. Med. 1993;30:438-446. doi: 10.1002/mrm.1910300406.

32. Pipe JG. Motion correction with PROPELLER MRI: Application to head motion and free-breathing cardiac imaging. Magn. Reson. Med. 1999;42:963-969. doi: 10.1002/(SICl)1522-2594(199911)42:5<963::AID-MRM17>3.0.CO;2-L.

33. Mehta BB, Ma D, Griswold MA. Image Registration and Robust Fitting for Motion Insensitive Magnetic Resonance Fingerprinting (MRF). In: Proceedings of International Society of Magnetic Resonance in Medicine. Singapore; 2016. p. 4256.

34. Yu Z, Zhao T, Assländer J, Lattanzi R, Sodickson DK, Cloos MA. Exploring the Sensitivity of Magnetic Resonance Fingerprinting to Different Types of Motion and Possible Correction Mechanisms. In: Proc. Intl. Soc. Mag. Reson. Med. Honolulu, HI; 2017. p. 3938. 
35. Xu Z, Lyu M, Hui E, Mei Y, Chen Z, Chen W, Wu EX, Feng Y. Motion Correction for Magnetic Resonance Fingerprinting by Using Sliding-Window Reconstruction and Image Registration. In: Proc. Intl. Soc. Mag. Reson. Med. Honolulu, HI; 2017. p. 1273.

36. Costagli M, Tosetti M, Donatelli G, Buonincontri G. Application of retrospective motion correction to magnetic resonance fingerprinting. In: Proc. Intl. Soc. Mag. Reson. Med. Honolulu, HI; 2017. p. 1315.

37. Cruz G, Botnar R, Prieto C. Motion corrected Magnetic Resonance Fingerprinting using Soft-weighted key-Hole (MRF-McSOHO). In: Proc. Intl. Soc. Mag. Reson. Med. Honolulu, HI; 2017. p. 935.

38. Strehl A, Ghosh J. Cluster Ensembles - A Knowledge Reuse Framework for Combining Multiple Partitions. J. Mach. Learn. Res. 2002;3:583-617.

39. Otsu N. A Threshold Selection Method from Gray-Level Histograms. IEEE Trans. Syst. Man. Cybern. 1979;9:62-66. doi: 10.1109/TSMC.1979.4310076.

40. Sahoo PK, Soltani S, Wong AKC, Chen YC. A survey of thresholding techniques. Comput. Vision, Graph. Image Process. 1988;41:233-260. doi: 10.1016/0734$189 \times(88) 90022-9$.

41. Mattes D, Haynor DR, Vesselle H, Lewellyn TK, Eubank W. Nonrigid multimodality image registration. In: Sonka M, Hanson KM, editors. Medical Imaging: Image Processing. SPIE Publications. ; 2001. pp. 1609-1620. doi: 10.1117/12.431046.

42. Raghunathan S, Stredney D, Schmalbrock P, Clymer BD. Image Registration Using Rigid Registration and Maximization of Mutual Information. In: The 13th Annual 
Medicine Meets Virtual Reality Conference. Long Beach, CA; 2005.

43. Pruessmann KP, Weiger $M$, Börnert $P$, Boesiger $P$. Advances in sensitivity encoding with arbitrary $k$-space trajectories. Magn. Reson. Med. 2001;46:638-651. doi: 10.1002/mrm.1241.

44. Fessler JA, Sutton BP. Nonuniform fast fourier transforms using min-max interpolation. IEEE Trans. Signal Process. 2003;51:560-574. doi: 10.1109/TSP.2002.807005.

45. Pierre EY, Ma D, Chen Y, Badve C, Griswold MA. Multiscale reconstruction for MR fingerprinting. Magn. Reson. Med. 2016;75:2481-2492. doi: 10.1002/mrm.25776.

46. Wang Z, Bovik AC, Sheikh HR, Simoncelli EP. Image Quality Assessment: From Error Visibility to Structural Similarity. IEEE Trans. Image Process. 2004;13:600-612. doi: 10.1109/TIP.2003.819861.

47. Lee JH, Hargreaves BA, Hu BS, Nishimura DG. Fast 3D imaging using variabledensity spiral trajectories with applications to limb perfusion. Magn. Reson. Med. 2003;50:1276-1285. doi: 10.1002/mrm.10644.

48. Spielman DM, Pauly JM, Meyer $\mathrm{CH}$. Magnetic resonance fluoroscopy using spirals with variable sampling densities. Magn. Reson. Med. 1995;34:388-394. doi: 10.1002/mrm.1910340316.

49. Fessler JA. https://web.eecs.umich.edu/ fessler/code/.

50. Myronenko A, Xubo Song. Intensity-Based Image Registration by Minimizing Residual Complexity. IEEE Trans. Med. Imaging 2010;29:1882-1891. doi: 
10.1109/TMI.2010.2053043.

51. Brown G, Pocock A, Zhao M-J, Lujan M. Conditional Likelihood Maximisation: A Unifying Framework for Information Theoretic Feature Selection Gavin Brown Adam Pocock Ming-Jie Zhao. J. Mach. Learn. Res. 2012;13:27-66.

52. Hamilton JI, Jiang Y, Ma D, Mehta BB, Lo W-C, Chen Y, Griswold MA, Seiberlich N. Cardiac MR fingerprinting for T1 and T2 mapping in four heartbeats. J. Cardiovasc. Magn. 2016;18:W1. doi: 10.1186/1532-429X-18-S1-W1.

53. Chen Y, Jiang Y, Pahwa S, Ma D, Lu L, Twieg MD, Wright KL, Seiberlich N, Griswold MA, Gulani V. MR Fingerprinting for Rapid Quantitative Abdominal Imaging. Radiology 2016;279:278-286. doi: 10.1148/radiol.2016152037.

54. Chen Y, Mehta BB, Hamilton JI, Ma D, Seiberlich N, Griswold MA, Gulani V. FreeBreathing 3D Abdominal Magnetic Resonance Fingerprinting Using Navigators. In: Proceedings of International Society of Magnetic Resonance in Medicine. Singapore; 2016. p. 716.

55. Wang Z, Li H, Zhang Q, Yuan J, Wang X. Magnetic Resonance Fingerprinting with compressed sensing and distance metric learning. Neurocomputing 2016;174:560-570. doi: 10.1016/j.neucom.2015.09.077.

56. Davies M, Puy G, Vandergheynst P, Wiaux Y. A Compressed Sensing Framework for Magnetic Resonance Fingerprinting. SIAM J. IMAGING Sci. 2014;7:2623-2656. doi: $10.1137 / 130947246$.

57. Zhao B, Setsompop K, Ye H, Cauley SF, Wald LL. Maximum Likelihood 
Reconstruction for Magnetic Resonance Fingerprinting. IEEE Trans. Med. Imaging 2016;35:1812-1823. doi: 10.1109/TMI.2016.2531640.

58. Doneva M, Amthor T, Koken P, Sommer K, Börnert P. Matrix completion-based reconstruction for undersampled magnetic resonance fingerprinting data. Magn. Reson. Imaging 2017;41:41-52. doi: 10.1016/J.MRI.2017.02.007.

59. Zhao B, Setsompop K, Adalsteinsson E, Gagoski B, Ye H, Ma D, Jiang Y, Ellen Grant P, Griswold MA, Wald LL. Improved magnetic resonance fingerprinting reconstruction with low-rank and subspace modeling. Magn. Reson. Med. 2017; early view. doi: 10.1002/mrm.26701.

60. Pierre EY, Griswold MA, Connelly A. Fast Analytical Solution for Extreme Unaliasing of MR Fingerprinting Image Series. In: Proceedings of International Society of Magnetic Resonance in Medicine. Honolulu, HI; 2017. p. 1353.

61. Hansen MS, Sørensen TS. Gadgetron: An open source framework for medical image reconstruction. Magn. Reson. Med. 2013;69:1768-1776. doi: $10.1002 / \mathrm{mrm} .24389$.

62. Ma D, Pierre EY, Jiang Y, Schluchter MD, Setsompop K, Gulani V, Griswold MA. Music-based magnetic resonance fingerprinting to improve patient comfort during MRI examinations. Magn. Reson. Med. 2016;75:2303-2314. doi: 10.1002/mrm.25818.

63. Ma D, Jiang Y, Chen Y, McGivney D, Mehta B, Gulani V, Griswold M. Fast 3D magnetic resonance fingerprinting for a whole-brain coverage. Magn. Reson. Med. 2017; early view. doi: 10.1002/mrm.26886. 
64. Liao C, Bilgic B, Manhard MK, Zhao B, Cao X, Zhong J, Wald LL, Setsompop K. 3D MR fingerprinting with accelerated stack-of-spirals and hybrid sliding-window and GRAPPA reconstruction. Neuroimage 2017;162:13-22. doi:

10.1016/J.NEUROIMAGE.2017.08.030.

65. Chen X, Yang Y, Cai X, Auger DA, Meyer CH, Salerno M, Epstein FH. Accelerated two-dimensional cine DENSE cardiovascular magnetic resonance using compressed sensing and parallel imaging. J. Cardiovasc. Magn. Reson. 2016;18. doi: 10.1186/s12968-016-0253-2.

66. Ma D, Coppo S, Chen Y, McGivney DF, Jiang Y, Pahwa S, Gulani V, Griswold MA. Slice profile and B1 corrections in 2D magnetic resonance fingerprinting. Magn. Reson. Med. 2017. doi: 10.1002/mrm.26580.

67. Ben-Eliezer N. Rapid and accurate T2 mapping from multi-spin-echo data using bloch-simulation-based reconstruction. Magn. Reson. Med. 2014;817:809-817. doi: 10.1002/mrm.25156.

68. Ehses P, Seiberlich N, Ma D, Breuer FA, Jakob PM, Griswold MA, Gulani V. IR TrueFISP with a golden-ratio-based radial readout: Fast quantification of T $1, T 2$, and proton density. Magn. Reson. Med. 2013;69:71-81. doi: 10.1002/mrm.24225.

69. Gloor M, Scheffler K, Bieri O. Quantitative magnetization transfer imaging using balanced SSFP. Magn. Reson. Med. 2008;60:691-700. doi: 10.1002/mrm.21705.

70. Crooijmans HJA, Scheffler K, Bieri O. Finite RF pulse correction on DESPOT2. Magn. Reson. Med. 2011;65:858-862. doi: 10.1002/mrm.22661. 


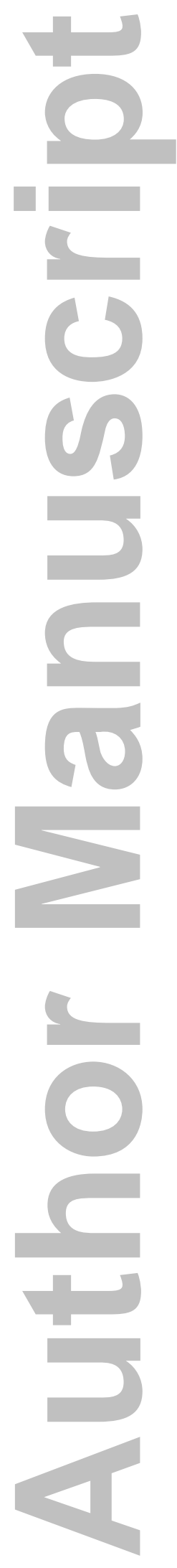

Magnetic Resonance in Medicine

This article is protected by copyright. All rights reserved. 


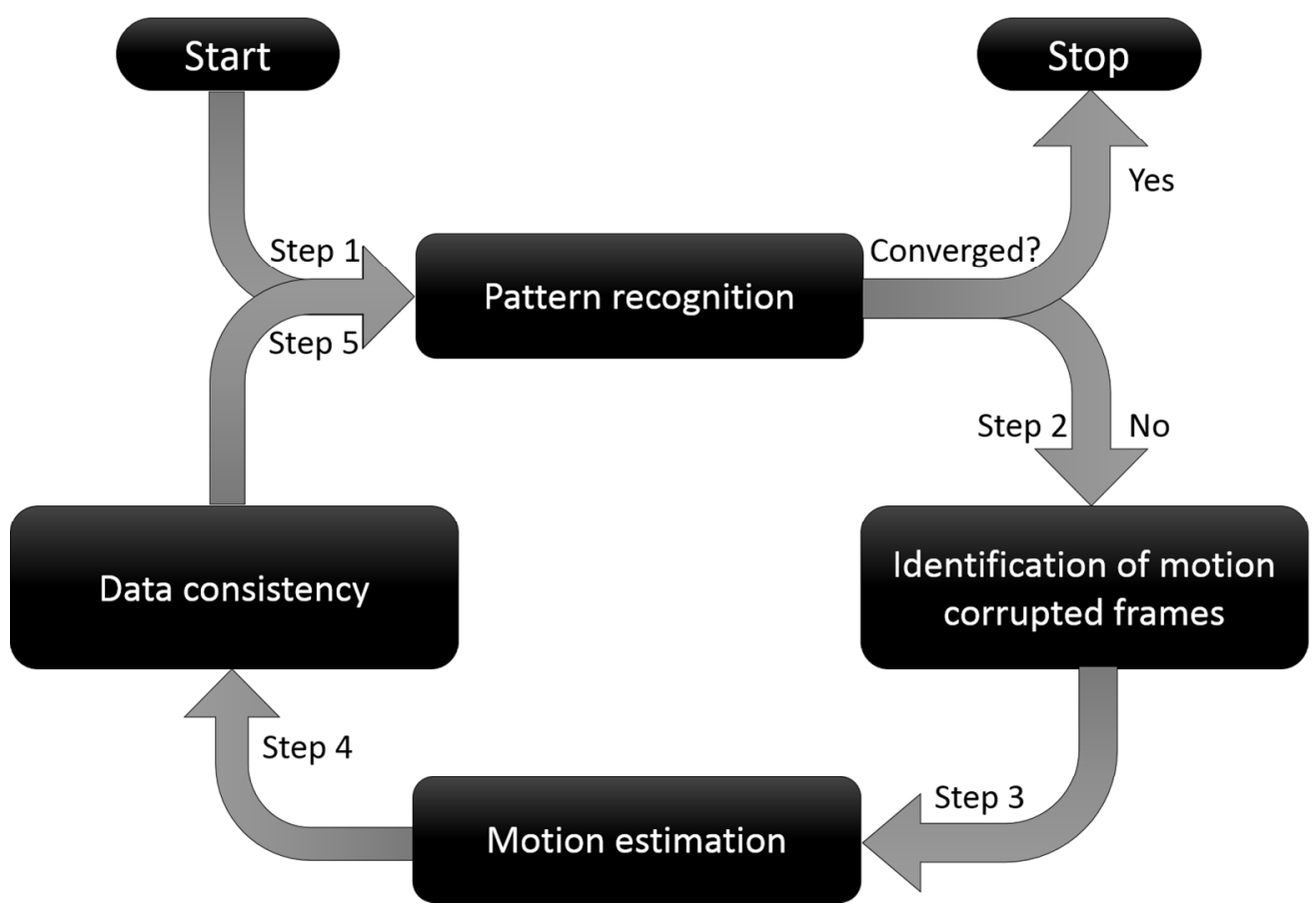

Figure 1: Flow chart of the motion insensitive magnetic resonance fingerprinting (MORF) algorithm. $175 \times 120 \mathrm{~mm}(300 \times 300 \mathrm{DPI})$ 


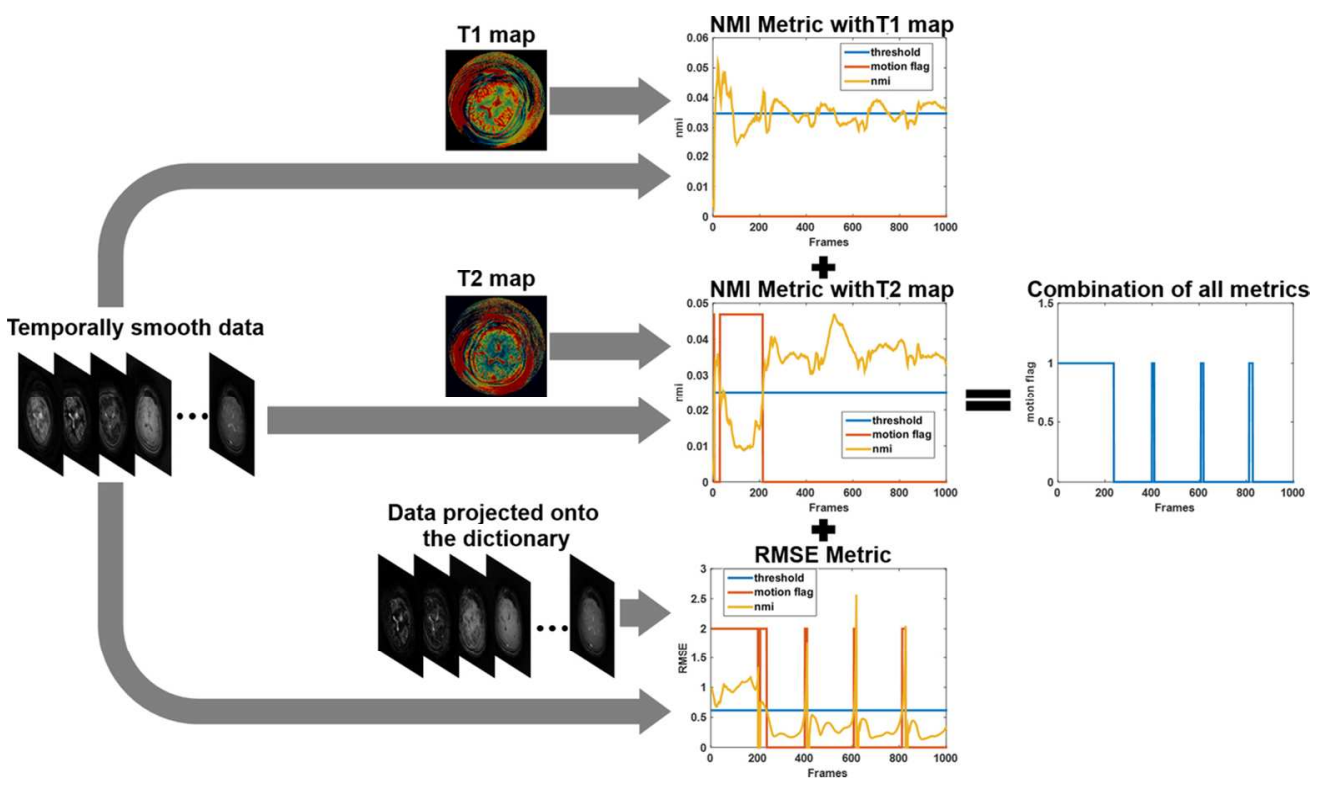

Figure 2: Steps involved in identification of motion corrupted frames. Motion corrupted frames are identified based on three metrics: Normalized mutual information (NMI) between each frame of the temporally smooth data and T1 map, NMI between each frame of the temporally smooth data and T2 map, and relative root mean square error (RMSE) between temporally smooth data and data projected onto the dictionary. Automatic thresholding is used to split each metric into two sections. The section with lower number frames is considered motion corrupted. For a given metric, if the number of identified motion corrupted frames are greater than one third of the total number of frames then those frames are discarded (top row). Finally, the results from the three metrics are combined using a logical OR operation to provide the motion flag (1: frame with motion ; 0: frame without motion) output.

$113 \times 66 \mathrm{~mm}(300 \times 300 \mathrm{DPI})$ 


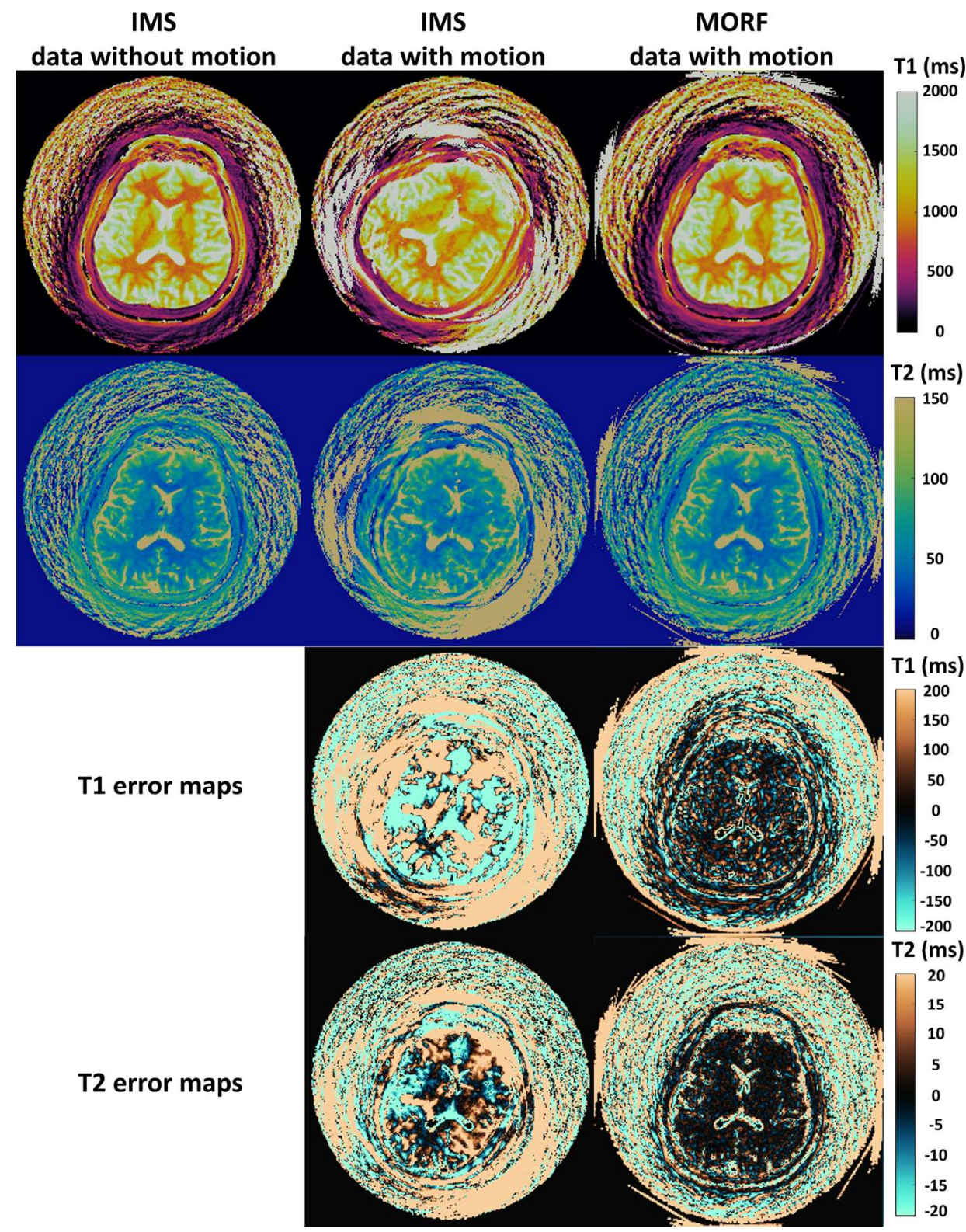

Figure 3: Example mapping results from the simulated single motion pattern in the beginning of the acquisition. First column: maps using IMS reconstruction from data without simulated motion; Second column: maps using IMS reconstruction from data with simulated single motion pattern; Third column: maps using MORF reconstruction from data with simulated single motion pattern. First row: T1 maps;

Second row: T2 maps; Third row: T1 error maps with first column as ground truth; Forth row: T2 error maps with first column as ground truth. IMS results present severe motion artifacts. However, MORF results closely resemble to the ground truth with minimal errors.

$177 \times 226 \mathrm{~mm}(300 \times 300 \mathrm{DPI})$ 


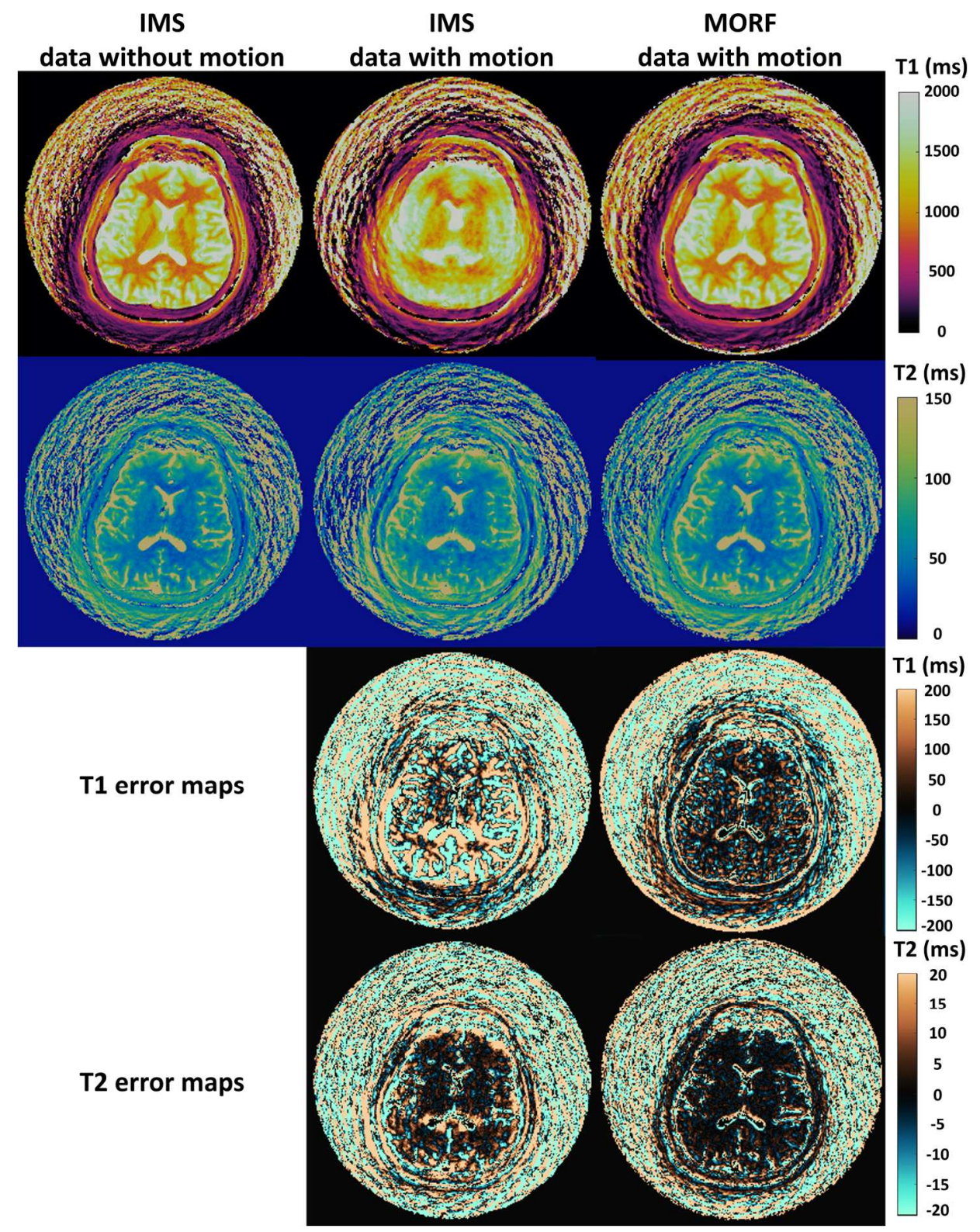

Figure 4: Example mapping results from the simulated oscillatory motion pattern in the beginning of the acquisition. First column: maps using IMS reconstruction from data without simulated motion; Second

column: maps using IMS reconstruction from data with simulated oscillatory motion pattern; Third column: maps using MORF reconstruction from data with simulated oscillatory motion pattern. First row: T1 maps; Second row: T2 maps; Third row: T1 error maps with first column as ground truth; Forth row: T2 error maps with first column as ground truth. Again, IMS results present motion artifacts which are significantly minimized in MORF results.

$177 \times 226 \mathrm{~mm}(300 \times 300 \mathrm{DPI})$ 


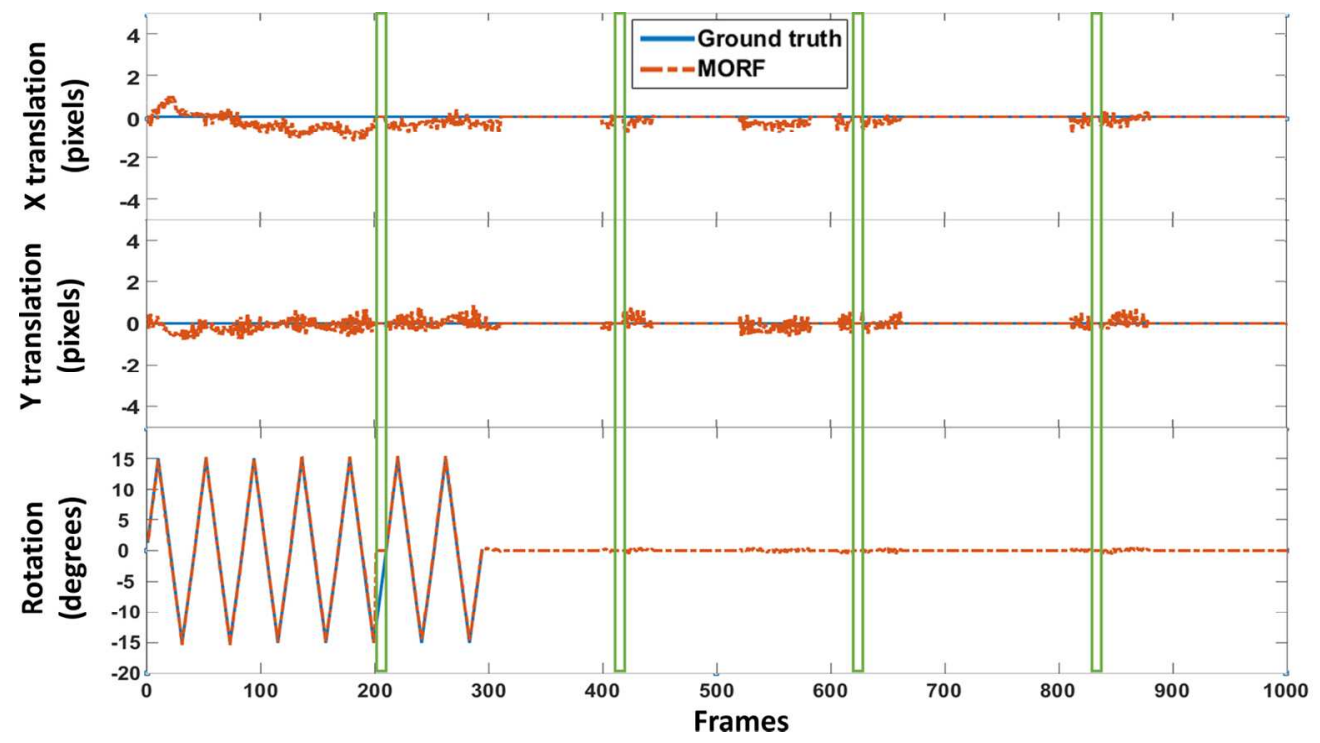

Figure 5: Accuracy of the estimation of rigid-body motion parameters. Example results from experiments with simulated oscillatory motion pattern in the beginning of the acquisition. top row: Translation along $X$. middle row: Translation along $Y$. bottom row: Rotation. The estimated rigid-body motion parameters are in close agreement with the ground truth values. The section highlighted with green blocks correspond to frames with zero flip angle where motion estimation is not performed.

$65 \times 36 \mathrm{~mm}(600 \times 600 \mathrm{DPI})$ 

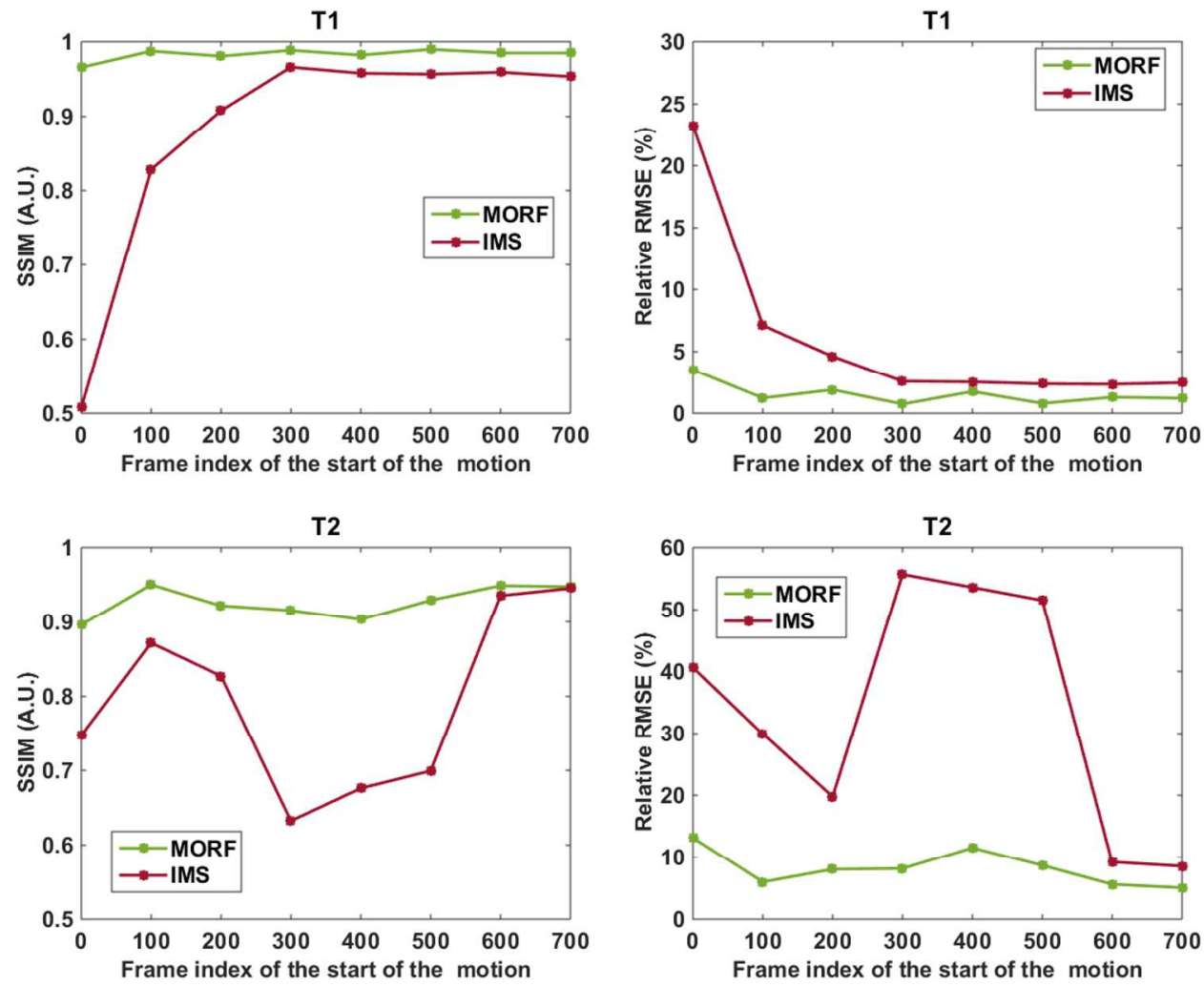

Figure 6: Quantitative results from set of simulations with single motion pattern evaluating the performance of the reconstruction algorithms to motion occurring at different stages of the acquisition. First column: SSIM results; Second column: Relative RMSE results; First row: Results for T1 values; Second

row: Results for T2 values. The variation in SSIM and rRMSE values for IMS algorithm illustrates the sensitivity of each parameter to motion for different parts of the acquisition. For MORF, the SSIM values are consistently high and the relative RMSE are consistently low, illustrating its agreement with ground truth for motion occurring at different stages of the acquisition.

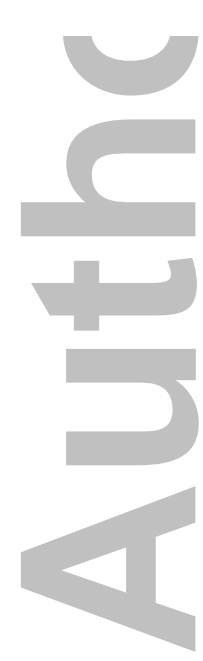

$$
138 \times 108 \mathrm{~mm}(600 \times 600 \mathrm{DPI})
$$


T1

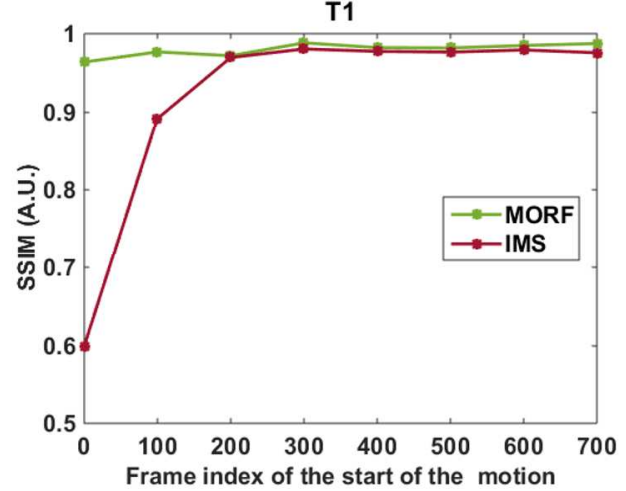

T2

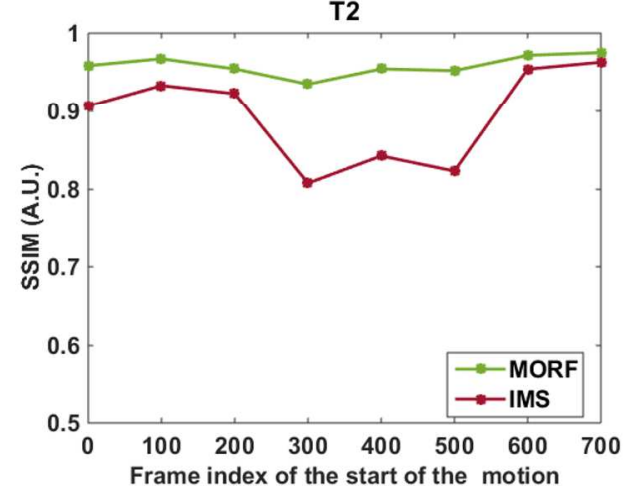

T1

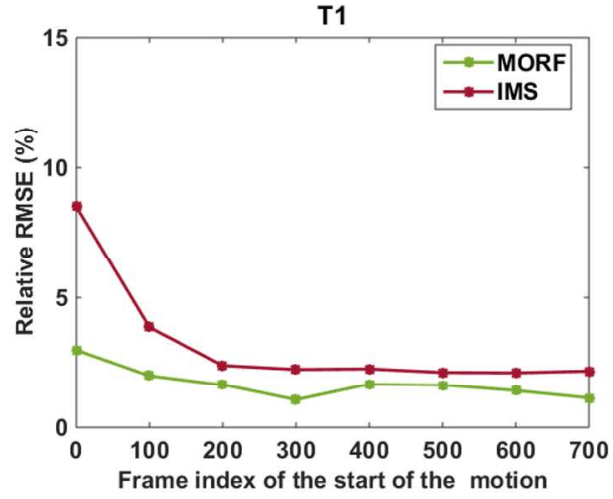

T2

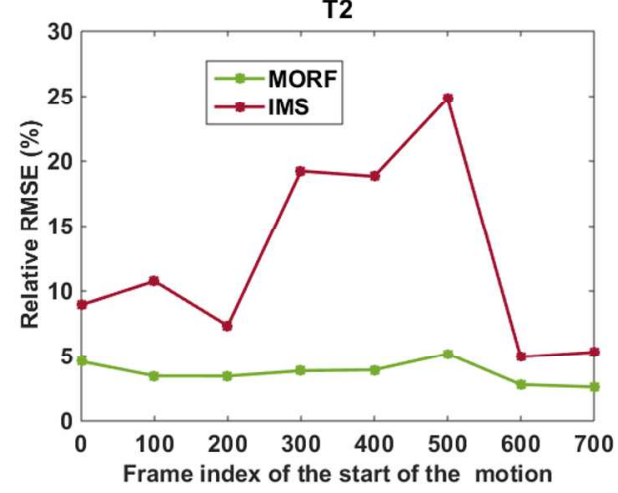

Figure 7: Quantitative results from set of simulations with oscillatory motion pattern evaluating the performance of the reconstruction algorithms to motion occurring at different stages of the acquisition. First column: SSIM results; Second column: Relative RMSE results; First row: Results for T1 values; Second row: Results for T2 values. Again, for all the datasets with motion during different parts of the acquisition, the SSIM and the relative RMSE values for MORF are consistently high and low, respectively.

$138 \times 108 \mathrm{~mm}(600 \times 600 \mathrm{DPI})$

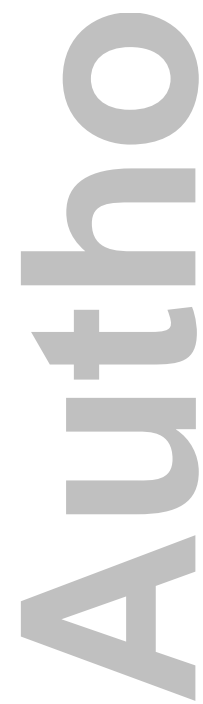



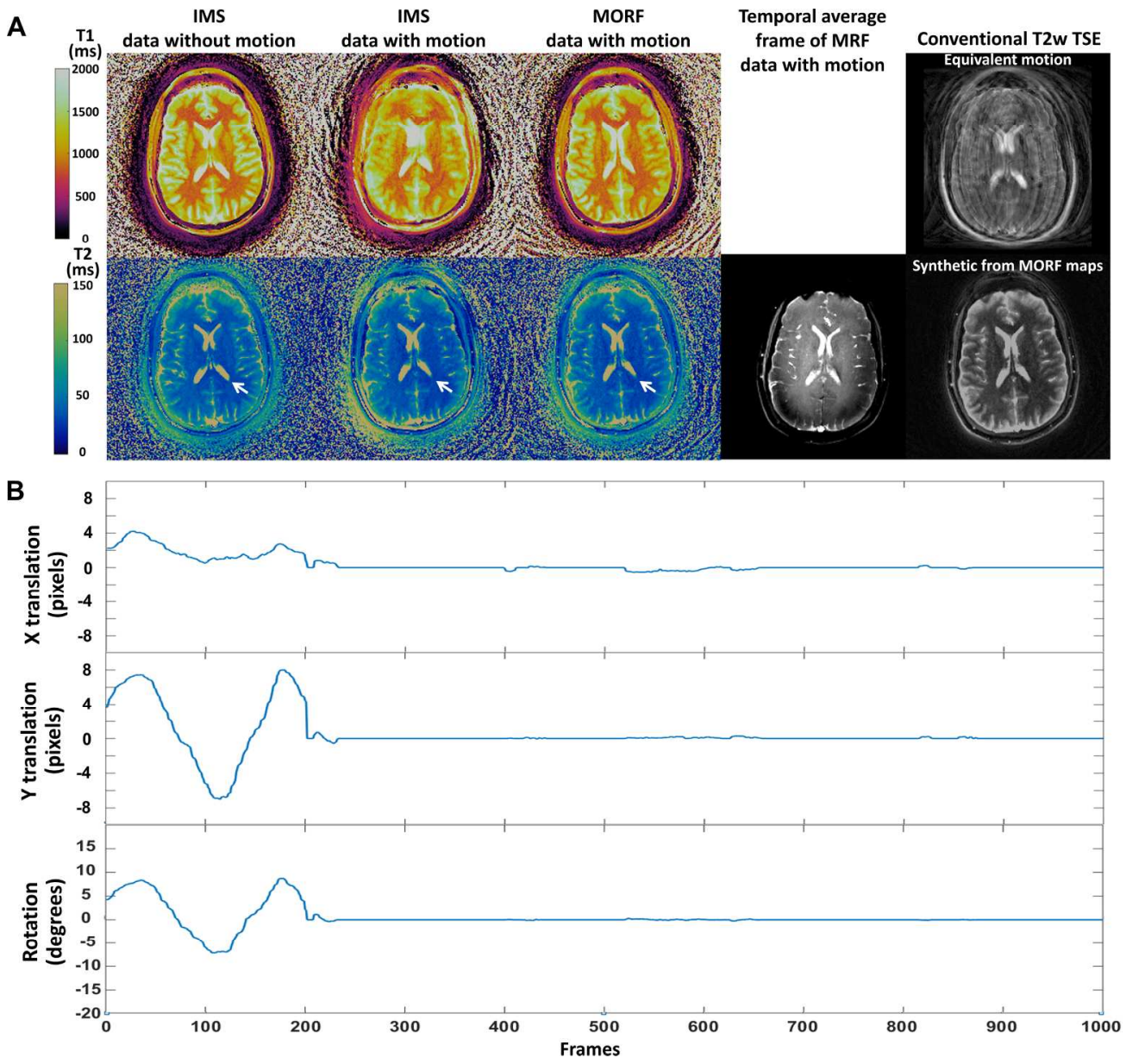

Figure 8: Results from prospectively motion corrupted in vivo experiment with motion in the initial $\sim 22 \%$ of the acquisition.

A: Reconstruction results. First column: IMS reconstruction maps from a separate scan without subject motion; Second column: IMS reconstruction maps using data with subject motion; Third column: MORF reconstruction maps using data with subject motion; Fourth column: Temporally averaged frame of the raw MRF data with subject motion; Fifth column: Conventional T2w-TSE. Top row is acquired data with equivalent subject motion and bottom row is synthetic image generated from maps reconstructed using

MORF from data with motion. The results are consistent with simulated motion experiments. MORF noticeably increases the robustness of the MRF acquisition towards subject motion. It should be noted that the discrepancy in the brain structure between data with and without motion might be due to change in the imaging location because of inter-scan subject motion.! + B: Estimated rigid-body motion parameters. top row: Translation along $X$; middle row: Translation along $Y$; bottom row: Rotation.

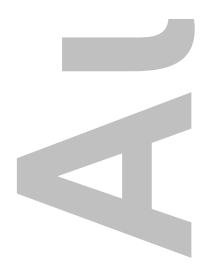

$211 \times 197 \mathrm{~mm}(300 \times 300 \mathrm{DPI})$ 
A

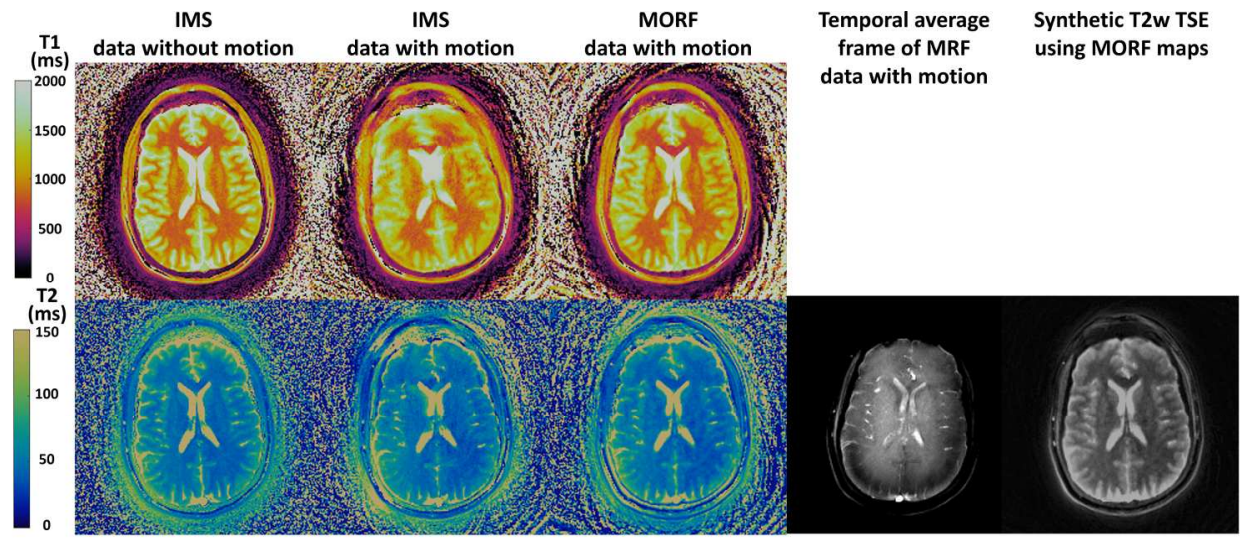

B

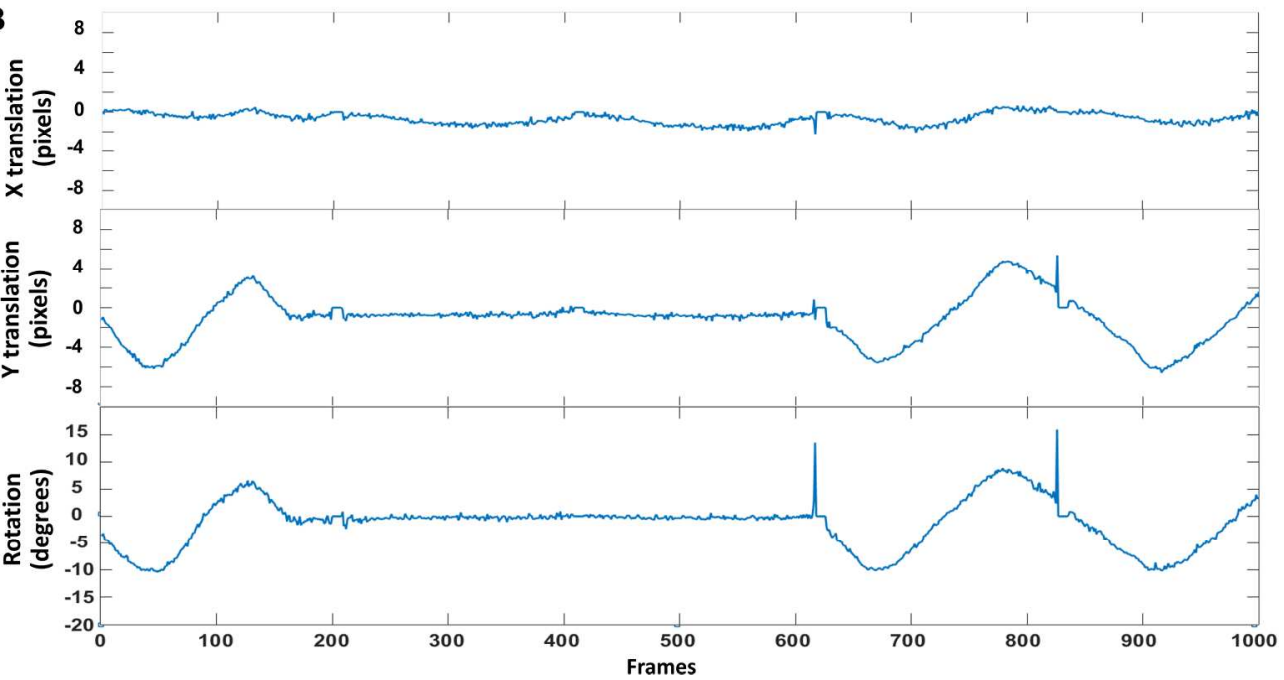

Figure 9: Results from a prospectively motion corrupted in vivo experiment. For this experiment the subject was requested to move randomly according to his/her will.

A: Reconstruction results. First column: IMS reconstruction maps from a separate scan without subject motion; Second column: IMS reconstruction maps using data with subject motion; Third column: MORF reconstruction maps using data with subject motion; Fourth column: Temporally averaged frame of the

raw MRF data with subject motion; Fifth column: Synthetic T2w-TSE image generated from maps reconstructed using MORF from data with motion. IMS results present a severe amount of motion artifacts not only in the T1 map but also in the T2 map. MORF results present significantly fewer artifacts compared to IMS and closely resemble the results from scan without motion.

B: Estimated rigid-body motion parameters. top row: Translation along $X$; middle row: Translation along $Y$; bottom row: Rotation. The subject ended up moving in the beginning $\sim 17 \%$ of the acquisition as well as at the ending $\sim 37 \%$ of the acquisition with a total of $\sim 54 \%$ data being motion corrupted.

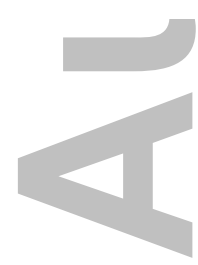

$211 \times 197 \mathrm{~mm}(300 \times 300 \mathrm{DPI})$ 


\section{Supporting Figures}
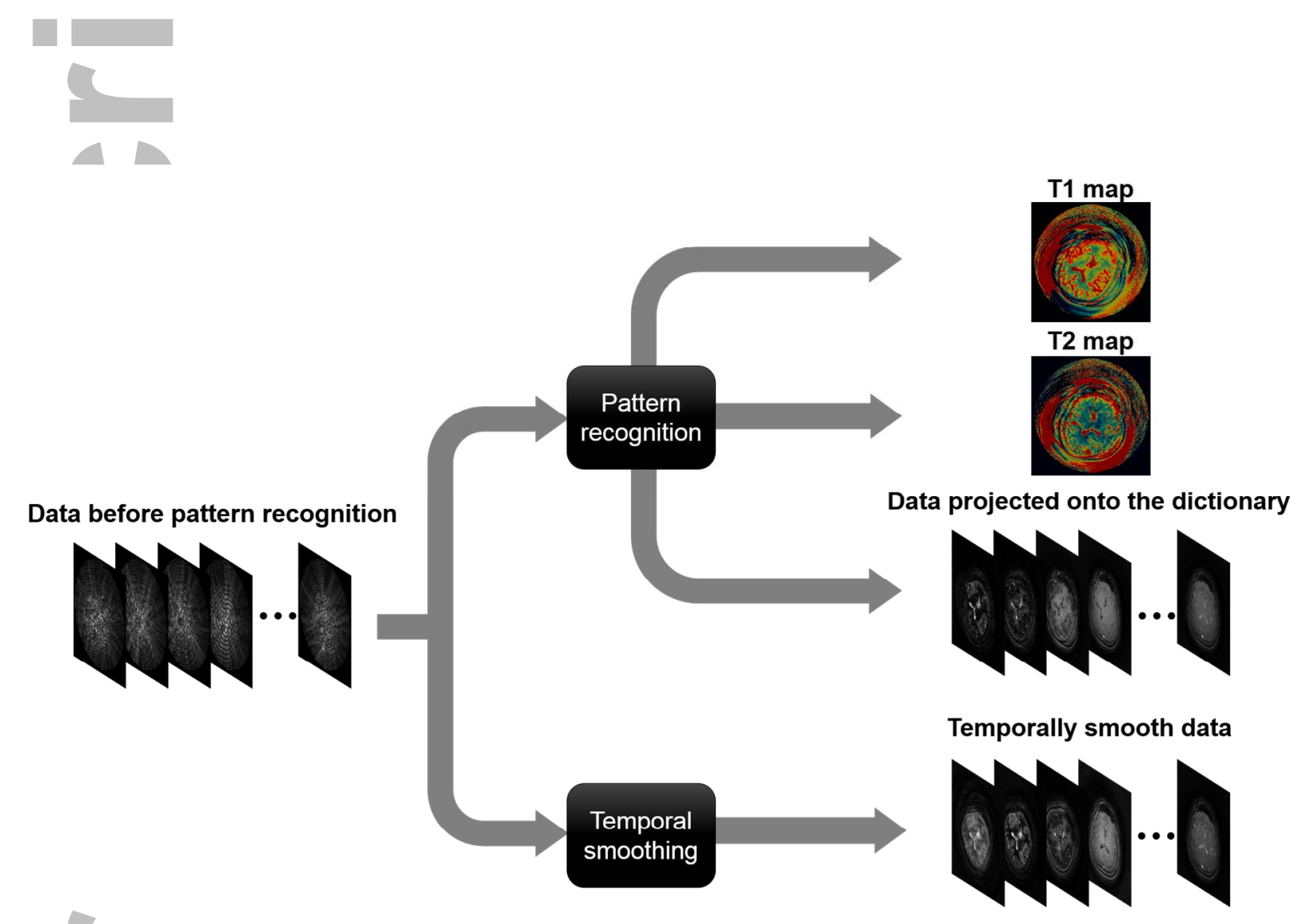

Supporting Figure S1: Origin of the components used for identification of motion corrupted frames.

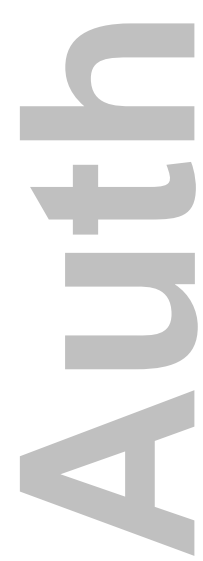



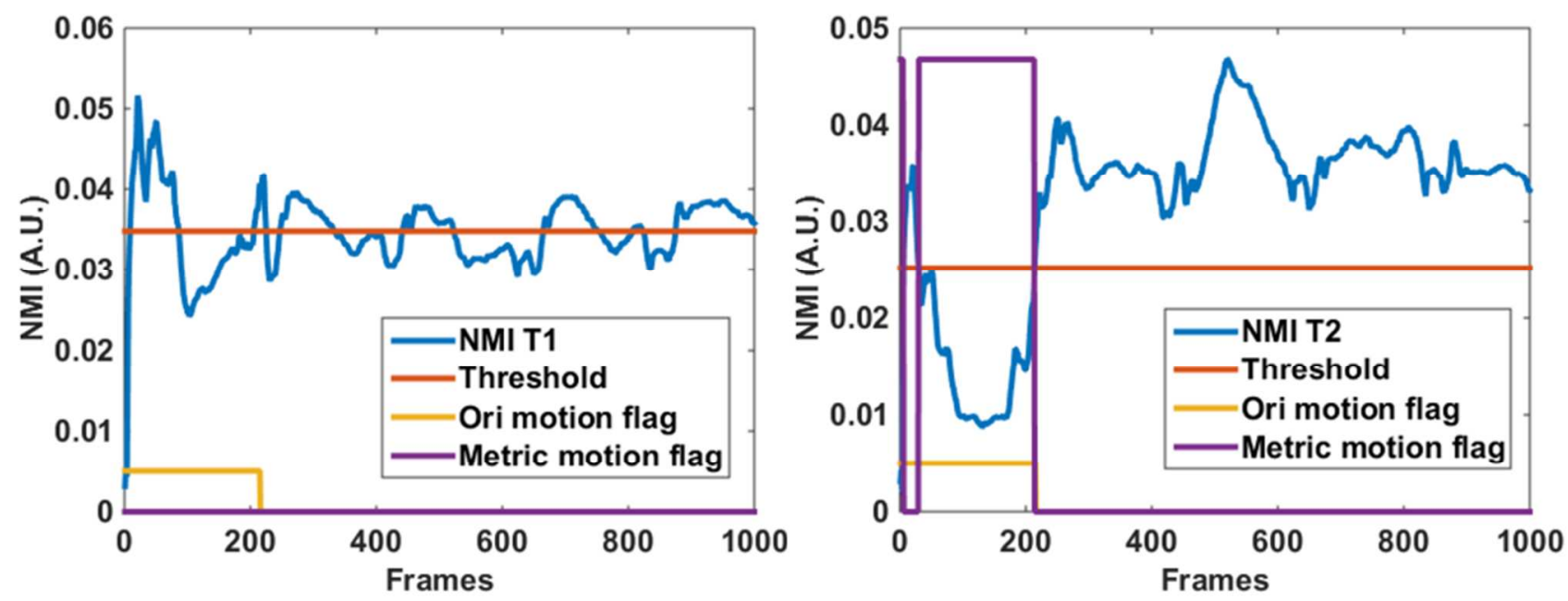

\section{Current T1 map}

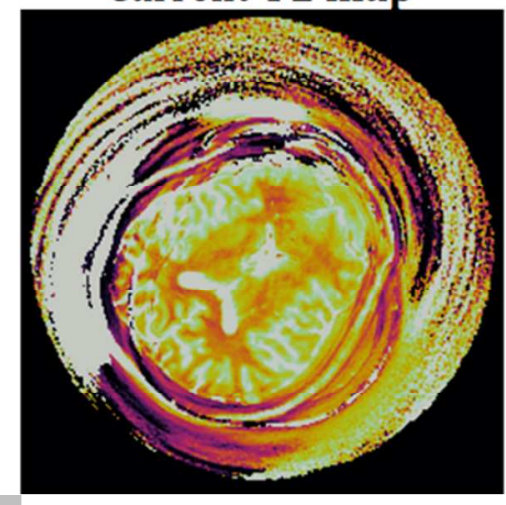

Current T2 map

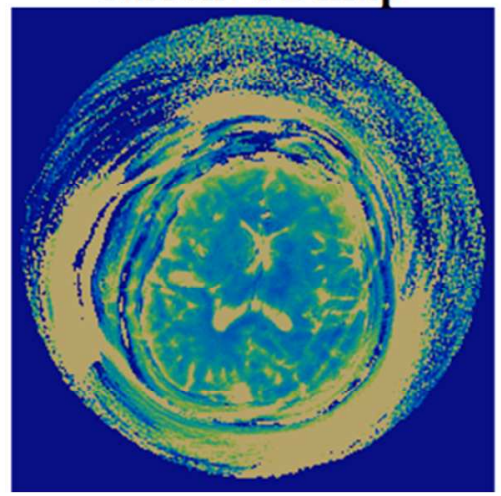

Supporting Figure S2: Example metric curves illustrating usefulness of NMI with T2 map metric. 

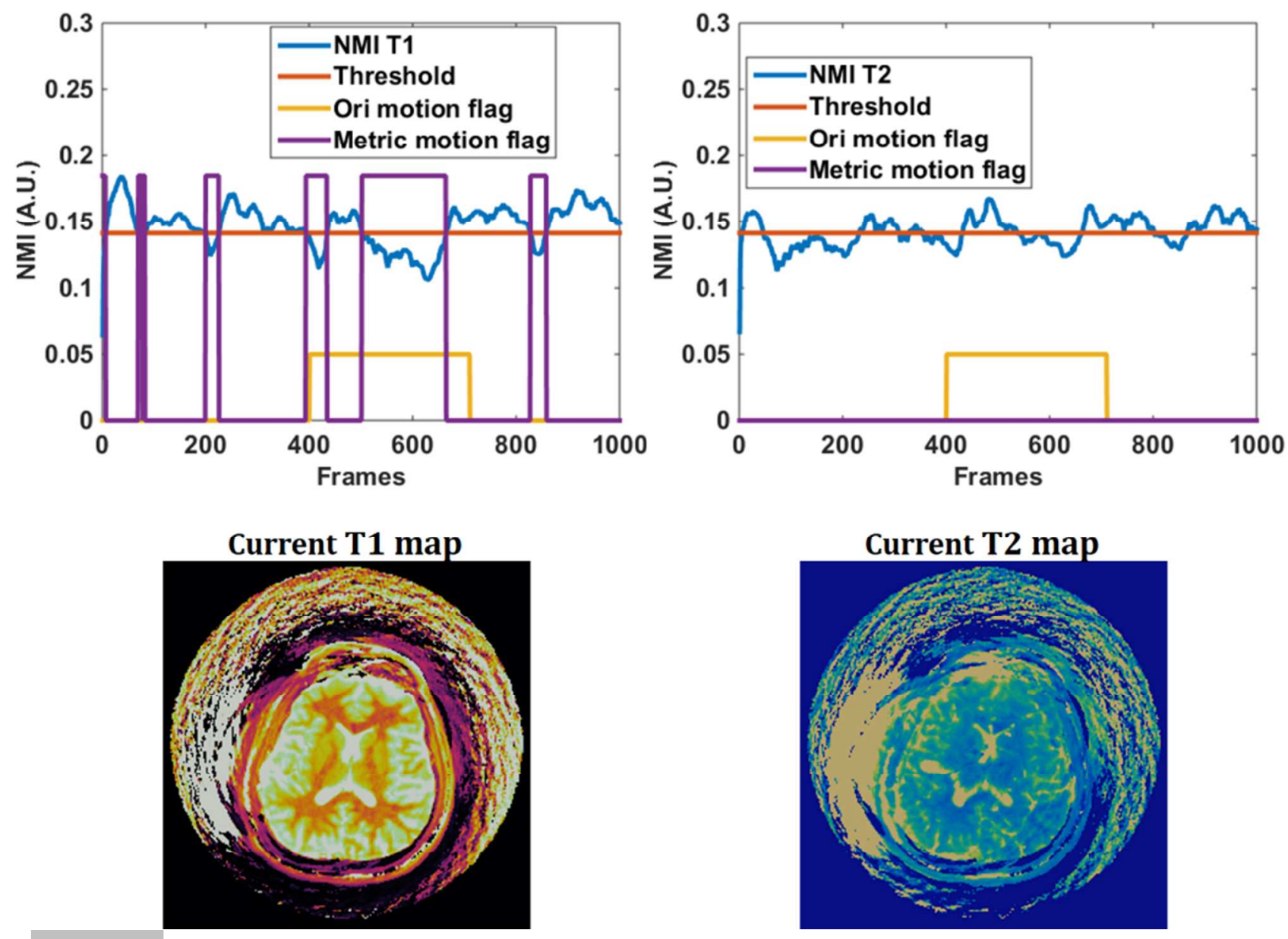

Supporting Figure S3: Example metric curves illustrating usefulness of NMI with T1 map metric.

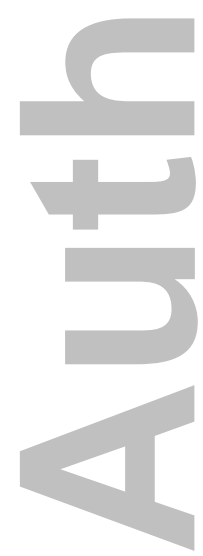



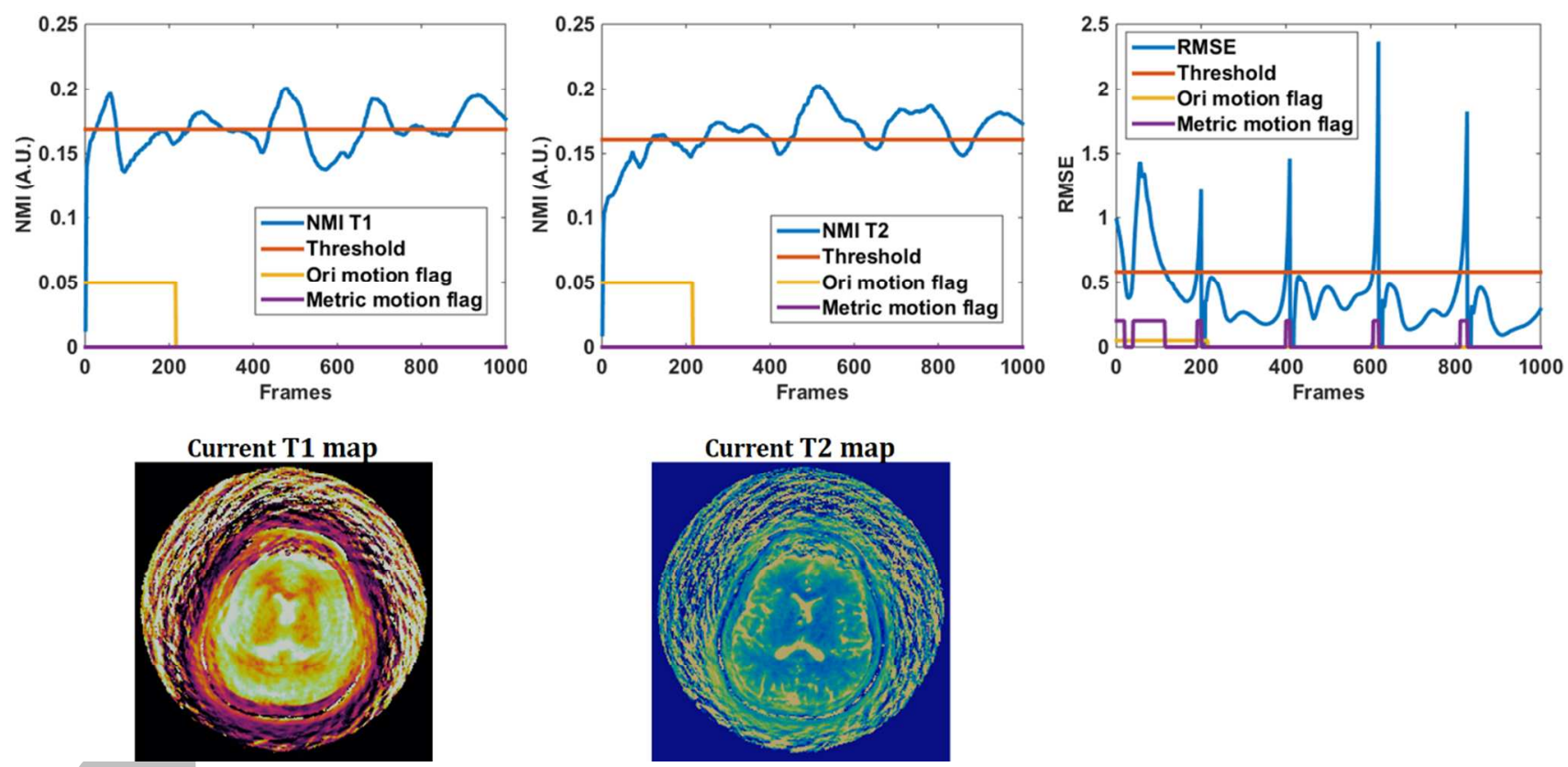

Supporting Figure S4: Example metric curves illustrating usefulness of RMSE metric. 


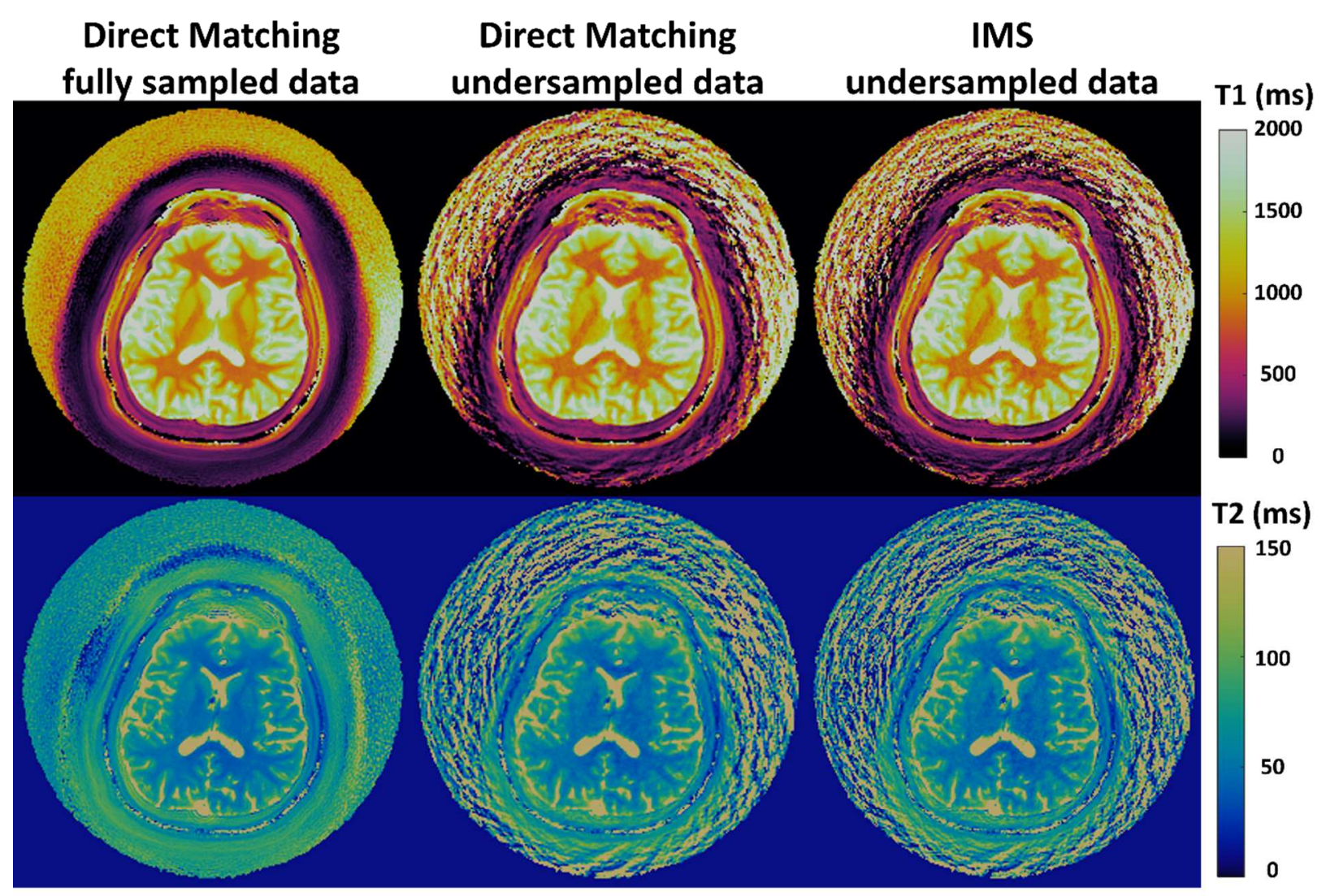

Supporting Figure S5: Comparison of fully sampled data with undersampled data and direct matching with IMS algorithm. We see a few differences between results using direct matching from fully sampled data (left) and results using direct matching from undersampled data (center). However, results using direct matching and IMS algorithm (right) from undersampled data are in close agreement. 
SCHOOL OF MEDICINE

December 132017

Matt A. Bernstein, Ph.D., Editor-in-Chief

Magnetic Resonance in Medicine

2030 Addison Street

Berkeley, CA 94704

Tel:+1(717)689-3694

\section{Dear Matt and the YIA Committee,}

I am writing today to support the nomination of Bhairav Mehta's paper entitled, "Image Reconstruction Algorithm for Motion Insensitive Magnetic Resonance Fingerprinting (MRF): MORF" for consideration for the ISMRM's Young Investigator Award. As a post-doc in my lab, Bhairav is perfectly qualified for the YIA. This work is almost entirely his... my only contribution was to say "maybe you can use the MRF time course to detect motion." He took this general idea and completely independently developed both a motion detection algorithm and an MRF reconstruction that can take advantage of this motion information. As shown in this study, the proposed MORF algorithm reconstructs quantitative parametric maps from a $13.5 \mathrm{~s}$ 2D brain MRF scan with minimal artifacts even if $54 \%$ data is motion corrupted. We feel that this will be a critical development in making MRF a true clinical reality even in patients with severe issues with motion, such as patients with Parkinson's Disease or other movement disorders and in children who may not be able to hold perfectly still during an exam. In a more general sense, I also believe that Bhairav will be a bright young scientist in our field, who will have a long career dedicated to increasing the value of MRI and making it available to everyone on the planet. I strongly urge you to grant him this award.

Thank you for your consideration!

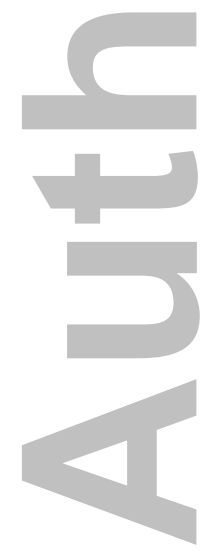

Sincerely,

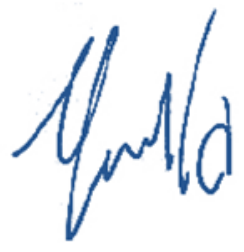

Mark A. Griswold, Ph.D.

MARK GRISWOLD, PH.D.

PROFESSOR OF RADIOLOGY, DIRECTOR OF MRI RESEARCH

CASE WESTERN RESERVE UNIVERSITY \& UNIVERSITY HOSPITALS OF CLEVELAND

MRI RESEARCH - BOLWELLB121, 11100 EUCLID AVENUE - CLEVELAND, OH・44106

EMAIL: MAG46@CASE.EDU PH: +1(216)844-8085FAX: +1(216)844-3062 


\section{University Library}

\section{- M M N E R VA A gateway to Melbourne's research publications}

Minerva Access is the Institutional Repository of The University of Melbourne

Author/s:

Mehta, BB;Ma, D;Pierre, EY;Jiang, Y;Coppo, S;Griswold, MA

Title:

Image reconstruction algorithm for motion insensitive MR Fingerprinting (MRF): MORF

Date:

2018-12-01

Citation:

Mehta, B. B., Ma, D., Pierre, E. Y., Jiang, Y., Coppo, S. \& Griswold, M. A. (2018). Image reconstruction algorithm for motion insensitive MR Fingerprinting (MRF): MORF. MAGNETIC RESONANCE IN MEDICINE, 80 (6), pp.2485-2500. https://doi.org/10.1002/mrm.27227.

Persistent Link:

http://hdl.handle.net/11343/283976 